\title{
Self-dual Einstein spaces, heavenly metrics, and twistors
}

\author{
Sergei Alexandrov, ${ }^{1, a)}$ Boris Pioline, ${ }^{2, b)}$ and Stefan Vandoren ${ }^{3, c)}$ \\ ${ }^{1}$ Laboratoire de Physique Théorique and Astroparticules, CNRS UMR 5207, Université \\ Montpellier II, 34095 Montpellier Cedex 05, France \\ ${ }^{2}$ Laboratoire de Physique Théorique et Hautes Energies, CNRS UMR 7589, Université \\ Pierre et Marie Curie, 4 Place Jussieu, 75252 Paris Cedex 05, France \\ ${ }^{3}$ Institute for Theoretical Physics and Spinoza Institute, Utrecht University, Leuvenlaan 4, \\ 3508 TD Utrecht, The Netherlands
}

(Received 15 January 2010; accepted 21 April 2010; published online 28 July 2010)

\begin{abstract}
Four-dimensional quaternion-Kähler metrics, or equivalently self-dual Einstein spaces $\mathcal{M}$, are known to be encoded locally into one real function $h$ subject to Przanowski's heavenly equation. We elucidate the relation between this description and the usual twistor description for quaternion-Kähler spaces. In particular, we show that the same space $\mathcal{M}$ can be described by infinitely many different solutions $h$, associated with different complex (local) submanifolds on the twistor space, and therefore to different (local) integrable complex structures on $\mathcal{M}$. We also study quaternion-Kähler deformations of $\mathcal{M}$ and, in the special case where $\mathcal{M}$ has a Killing vector field, show that the corresponding variations in $h$ are related to eigenmodes of the conformal Laplacian on $\mathcal{M}$. We exemplify our findings on the four-sphere $S^{4}$, the hyperbolic plane $H^{4}$, and on the "universal hypermultiplet," i.e., the hypermultiplet moduli space in type IIA string compactified on a rigid CalabiYau threefold. (C) 2010 American Institute of Physics. [doi:10.1063/1.3430574]
\end{abstract}

\section{INTRODUCTION AND SUMMARY}

Einstein spaces with self-dual Weyl curvature have been much studied both in the mathematics and physics literature. On the mathematics side, they embody the notion of quaternion-Kähler geometry in four dimensions and are thus amenable to the same algebrogeometric, twistorial techniques as their higher-dimensional cousins. ${ }^{1}$ In the case of compact Einstein spaces with positive scalar curvature, the self-duality constraint is so strong as to leave only two possibilities: the four-sphere $S^{4}$ equipped with the round metric, and the complex projective plane $C P^{2}$ $=\mathrm{SU}(3) / \mathrm{U}(2)$ equipped with the Fubini-Study metric. For nonpositive curvature, however (the zero-curvature case corresponding to hyperkähler manifolds), the situation is much richer and a classification is still lacking.

On the physics side, self-dual Einstein spaces are the natural notion of gravitational instanton in the presence of a cosmological constant. In the case of negative curvature, these spaces can also be used as target spaces for locally supersymmetric sigma models with eight supercharges. ${ }^{2} \mathrm{~A}$ prominent example is the hypermultiplet moduli space $\mathcal{M}_{H}(X)$ for type IIA string theory compactified on a rigid Calabi-Yau manifold $X$, which has received much attention in recent years. ${ }^{3-12}$ Computing the exact self-dual Einstein metric on $\mathcal{M}_{H}(X)$ including all instanton corrections is still an open problem, which motivates the present study.

As shown long ago by Przanowski, ${ }^{13}$ self-dual Einstein metrics $\mathcal{M}$ can be described locally by one real function $h$ on an open neighborhood $\mathrm{U}$ in $\mathcal{M}$ coordinatized by complex coordinates $z^{\alpha}$, $\alpha=1,2$, subject to the partial differential equation,

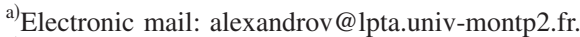

${ }^{b}$ Electronic mail: pioline@1pthe.jussieu.fr.

${ }^{c)}$ Electronic mail: s.j.g.vandoren@uu.nl.
} 


$$
\operatorname{Prz}(h) \equiv h_{1 \overline{1}} h_{2 \overline{2}}-h_{12} h_{12}^{-}+\left(2 h_{1 \overline{1}}-h_{1} h_{1}^{-}\right) \mathrm{e}^{h}=0,
$$

where $h_{1} \equiv \partial h / \partial z^{1}$, etc. Any solution of this equation then leads to a self-dual Einstein metric on $\mathrm{U}$ given by

$$
\mathrm{d} s_{\mathcal{M}}^{2}=-\frac{6}{\Lambda}\left(h_{\alpha \bar{\beta}} \mathrm{d} z^{\alpha} \mathrm{d} z^{\bar{\beta}}+2 \mathrm{e}^{h}\left|\mathrm{~d} z^{2}\right|^{2}\right) \equiv 2 g_{\alpha \bar{\beta}} \mathrm{d} z^{\alpha} \mathrm{d} z^{\bar{\beta}},
$$

normalized such that the Ricci scalar satisfies the standard convention in four dimensions $R$ $=4 \Lambda$. "Master equation" (1) is a variant of Plebanski's "first heavenly equation," which similarly parametrizes hyper-Kähler metrics in terms of one real function on an open set in $\mathrm{C}^{2}$. It is crucial to note that (2) exhibits the metric in Hermitian form with respect to a local (integrable) complex structure $J_{h}$ with complex coordinates $z^{1}, z^{2}$. This, in general, can only hold locally: indeed, self-dual Einstein spaces generally have no globally defined complex structures, as exemplified by $S^{4}$.

The Przanowski form of metric (2) provides a concise way of summarizing the constraints of quaternion-Kähler geometry in four dimensions. Moreover, it offers a convenient starting point for analyzing perturbations of $\mathcal{M}$ consistent with the self-dual Einstein property: ${ }^{11}$ indeed, such perturbations correspond to solutions $\delta h$ of the "linearized master equation,"

$$
\mathrm{dPrz}_{h}(\delta h)=0,
$$

where $h$ is a solution of (1), $\delta h$ its infinitesimal variation, and we defined the linearized Przanowski operator,

$$
\mathrm{dPrz}_{h} \equiv\left(h_{2 \overline{2}}+2 \mathrm{e}^{h}\right) \partial_{1} \partial_{1}^{-}+h_{11}^{-} \partial_{2} \partial_{2}^{-}-h_{12} \partial_{2} \partial_{1}^{-}-h_{21}^{-} \partial_{1} \partial_{2}^{-}+\mathrm{e}^{h}\left(2 h_{1 \overline{1}}-\left|h_{1}\right|^{2}-h_{1} \partial_{1}^{-}-h_{1}^{-} \partial_{1}\right) .
$$

Of course, there may be obstructions to integrating a solution of (4) to a smooth deformation of $\mathcal{M}$, as must be the case for compact positively curved manifolds. Moreover, as will be apparent presently, some of the solutions of (4) may be "pure gauge," i.e., may describe the same metric $\mathcal{M}$ up to diffeomorphism.

Indeed, there are, in general, infinitely many ways of expressing a given self-dual Einstein metric in Przanowski form (2). One obvious source of infinities comes from holomorphic changes of variables of the restricted form, ${ }^{14}$

$$
\begin{gathered}
z^{1} \rightarrow z^{\prime 1}=f\left(z^{1}, z^{2}\right), \quad z^{2} \rightarrow z^{\prime 2}=g\left(z^{2}\right), \\
h\left(z^{1}, z^{2}\right) \rightarrow h^{\prime}\left(z^{1}, z^{2}\right)=h\left(f\left(z^{1}, z^{2}\right), g\left(z^{2}\right)\right)-\log \left(g_{2}\left(z^{2}\right) \bar{g}_{2}^{-}\left(\bar{z}^{2}\right)\right) .
\end{gathered}
$$

This change of variables preserves the local complex structure $J_{h}$ and leads to the same metric up to diffeomorphism. At the infinitesimal level, the holomorphic change of variables (5) leads to "pure gauge" solutions of linearized Eq. (4) of the form

$$
\mathrm{dPrz}_{h}\left(h_{1} \delta f+h_{2} \delta g+\delta g_{2}\right)=\left(\delta f_{1}+\delta f \partial_{1}+\delta g_{2}+\delta g \partial_{2}\right) \operatorname{Prz}(h)=0,
$$

where $\delta f\left(z^{1}, z^{2}\right)$ and $\delta g\left(z^{2}\right)$ are infinitesimal versions of (5).

More significantly, as emphasized by $\operatorname{Tod}^{15}$ and reviewed later in this work, Przanowski ansatz (2) can be reached for any choice of local integrable complex structure $J$. In particular, different local integrable complex structures $J$ and $J^{\prime}$ lead to different solutions $h\left(z^{1}, z^{2}\right)$ and $h^{\prime}\left(z^{\prime 1}, z^{\prime 2}\right)$ of the master equation, such that $J=J_{h}$ and $J^{\prime}=J_{h^{\prime}}$. These solutions describe the same self-dual Einstein metric, but the coordinate systems $\left(z^{1}, z^{2}\right)$ and $\left(z^{\prime 1}, z^{\prime 2}\right)$ are, in general, related by a nonholomorphic change of variables, as depicted in Fig. 1 (to be elaborated on below). Quaternion-Kähler spaces admit infinitely many integrable local complex structures, which provides infinitely many ways of expressing the same self-dual Einstein metric in Przanowski's form. Unfortunately, it is not known how to express the corresponding "pure gauge" solutions $\delta h$ of (3) in terms of the unperturbed solution $h$ and its derivatives as in (7). 


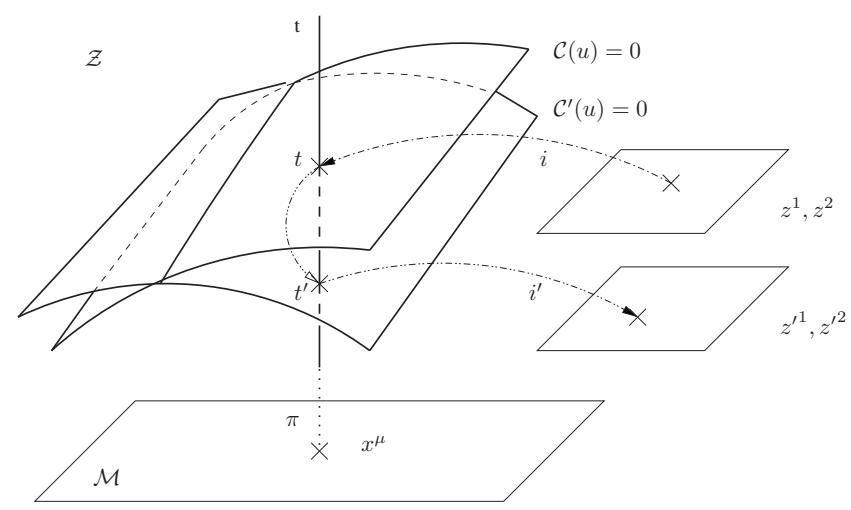

FIG. 1. Two Przanowski coordinate systems $\left(z^{1}, z^{2}\right),\left(z^{\prime 1}, z^{\prime 2}\right)$ associated with two different submanifolds $\mathcal{C}, \mathcal{C}^{\prime} \subset \mathcal{Z}$ are related by a nonholomorphic coordinate change. The relation is found by intersecting the twistor line through $i\left(z^{1}, z^{2}\right)$ $\in \mathcal{C}$ (the vertical line in this figure) with $\mathcal{C}^{\prime}$.

Our purpose in this note is to make contact between the Przanowski parametrization of self-dual Einstein spaces, reviewed above, and the standard twistor description of quaternionKähler spaces, recalled below. In doing so, we shall address the problem just raised and provide a way to generate all solutions of the master equation which describe the same self-dual Einstein space using twistor techniques.

\section{A. Preview of main results}

To preview our main result, recall that for any $4 n$-dimensional quaternion-Kähler space $\mathcal{M}$, one may construct its twistor space $\mathcal{Z}$, a two-sphere bundle over $\mathcal{M}$ which admits a canonical integrable complex structure together with a complex contact structure. Any local section of this bundle gives rise to an almost complex structure on an open subset $U=\pi(\mathcal{U}) \subset \mathcal{M}$. The key fact for us is that this (local) almost complex structure is integrable if and only if the submanifold $\mathcal{C}$ defined by the section is a (local) complex submanifold on $\mathcal{U} \subset \mathcal{Z}^{16}$

On the other hand, the quaternion-Kähler metric on $\mathcal{M}$ is related to the Kähler-Einstein metric on $\mathcal{Z}$ via

$$
\frac{\Lambda}{12} \mathrm{~d} s_{\mathcal{M}}^{2}=\mathrm{d} s_{\mathcal{Z}}^{2}-\mathrm{e}^{-2 K}|\mathcal{X}|^{2}
$$

where $\mathcal{X}$ is the (local) complex contact one-form and $K$ is the (local) Kähler potential on $\mathcal{Z}$. Restricting (8) to the submanifold $\mathcal{C}$ and identifying $h_{\mathcal{C}}=-2 K$ on that slice, we can express the quaternion-Kähler metric on $\mathcal{M}$ in the Hermitian form, ${ }^{17}$

$$
\mathrm{d} s_{\mathcal{M}}^{2}=-\frac{6}{\Lambda}\left(\partial_{\alpha} \partial_{\bar{\beta}} h_{\mathcal{C}} \mathrm{d} z^{\alpha} \mathrm{d} \bar{z}^{\bar{\beta}}+2 \mathrm{e}^{h_{\mathcal{C}}}\left|\mathcal{X}_{\mathcal{C}}\right|^{2}\right)
$$

where $z^{\alpha}, \alpha=1, \ldots, 2 n$ are complex coordinates along $\mathcal{C}$ and $\mathcal{X}_{\mathcal{C}}$ is the restriction of the contact one-form to $\mathcal{C}$. For $n=1$, one may always choose complex coordinates $z^{1}, z^{2}$ and perform a suitable Kähler transformation, such that $\left.\mathcal{X}\right|_{\mathcal{C}}=\mathrm{d} z^{2}$, hence recovering Przanowski ansatz (2). As for master equation (1), it can be shown to follow from the Monge-Ampère equation ${ }^{18}$ on $\mathcal{Z}$. We have therefore identified the twistorial origin of the Przanowski function $h_{\mathcal{C}}$ : it is just a "tomogram" of the Kähler potential $K$ along the complex submanifold $\mathcal{C}$. Varying the choice of locally integrable complex structure $J$ on $\mathcal{M}$, therefore the complex submanifold $\mathcal{C}$ of $\mathcal{Z}$, one can in principle reconstruct the Kähler potential on the whole of $\mathcal{Z}$.

Having established the twistorial origin of the Przanowski function, we can cast the solutions of linearized master equation (3) into the formalism developed in our previous work ${ }^{19}$ for linear deformations of quaternion-Kähler manifolds. To this end, we use the fact from Ref. 19 that the 
deformations of the Kähler potential $K$ on $\mathcal{Z}$ [or rather, of the contact potential, defined in (14) and (17) below] can be written as a contour integral of a certain holomorphic function [more precisely a section $H_{(1)}$ of the Čech cohomology group $\left.H^{1}(\mathcal{Z}, \mathcal{O}(2))\right]$, which encodes the variation in the contact transformations between various local Darboux coordinate systems on $\mathcal{Z}$. In the special case where the unperturbed metric $\mathcal{M}$ has a Killing vector, this leads to contour integral formula (142) for the corresponding deformation $\delta h$ of the Przanowski function. This formula is very similar to Penrose-type formula (139) which produces eigenmodes of the conformal Laplacian from sections $\Psi$ in $H^{1}(\mathcal{Z}, \mathcal{O}(-2))$ (as discussed in Ref. 20). This is not an accident because, when $\mathcal{M}$ has a Killing vector, linearized master equation (3) turns out to be equivalent to the conformal Laplace equation for the rescaled function $\delta h /\left|h_{1}\right|^{2}$, as explained in (133) below. In this fashion, we can identify the holomorphic sections $H_{(1)}$ and $\Psi$ in the particular trivialization introduced in Ref. 20.

The rest of this note aims at substantiating the above claims and illustrating them on various examples. It is organized as follows. In Sec. II, we review the twistorial description of quaternionKähler spaces. In Sec. III, we explain how all solutions of the master equation can be obtained by restricting the Kähler potential on the twistor space on any local complex submanifold, provide explicit formulas for relating different classes of solutions, and discuss how to recover the full twistor space from a single "tomogram." In Sec. IV we consider self-dual Einstein spaces with one or two commuting isometries and relate the respective Toda and Calderbank-Pedersen descriptions to the Przanowski framework. In Sec. V we illustrate these results by revisiting the $S^{4}$ and $H^{4}$ examples, as well as a deformed, noncompact version of $\mathrm{C} P^{2}$ which describes the perturbative hypermultiplet moduli space of type IIA string theory compactified on a rigid Calabi-Yau threefold. Finally, in Sec. VI, we present one of our main results, a Penrose-type formula which produces a solution to linearized master equation (3) from an element of $H^{1}(\mathcal{Z}, \mathcal{O}(-2))$ (locally, a holomorphic function on $\mathcal{Z}$ ) and explain the relation with the $H^{1}(\mathcal{Z}, \mathcal{O}(2))$ section which governs the perturbations of $\mathcal{Z}$. These results are illustrated on instanton corrections to the aforementioned hypermultiplet moduli space.

\section{REVIEW OF THE TWISTOR APPROACH TO QUATERNIONIC GEOMETRIES}

In real dimension $4 n \geq 8$, a quaternion-Kähler manifold $\mathcal{M}$ is a Riemannian manifold with metric $g_{\mathcal{M}}$ and Levi-Civita connection $\nabla$, such that the holonomy group is contained in $\operatorname{USp}(n)$ $\times \mathrm{SU}(2)$, see, e.g., Ref. 21. In dimension $4 n=4$, the case of interest in this paper, this holonomy condition is trivially satisfied and must be replaced by the condition that the metric be Einstein and the Weyl curvature be self-dual. In any dimension, quaternion-Kähler manifolds can also be defined as Riemannian manifolds that admit a triplet of almost complex Hermitian structures $\vec{J}$ [defined up to $\mathrm{SU}(2)$ rotations] satisfying the algebra of the unit imaginary quaternions. The quaternionic two-forms $\vec{\omega}_{\mathcal{M}}(X, Y) \equiv g_{\mathcal{M}}(\vec{J} X, Y)$ must be covariantly closed with respect to the $\mathrm{SU}(2)$ part $\vec{p}$ of the Levi-Civita connection and be proportional to the curvature of $\vec{p}$,

$$
\mathrm{d} \vec{\omega}_{\mathcal{M}}+\vec{p} \times \vec{\omega}_{\mathcal{M}}=0, \quad \mathrm{~d} \vec{p}+\frac{1}{2} \vec{p} \times \vec{p}=\frac{\nu}{2} \vec{\omega}_{\mathcal{M}},
$$

where we use the notation $(\vec{v} \times \vec{w})^{a}=\epsilon^{a b c} v^{b} \wedge w^{c} ; a, b, \ldots=1,2,3$. The fixed coefficient $\nu$ is related to the constant Ricci scalar curvature via $R=4 n(n+2) \nu$ and therefore in four dimensions, $\nu$ $=\Lambda / 3$ with the standard convention for the cosmological constant $\Lambda$ as in Sec. I.

\section{A. Twistor space and complex contact structure}

A quaternion-Kähler manifold $(\mathcal{M}, g, \vec{J})$ can be efficiently described by means of its twistor space $\mathcal{Z}$, a $C P^{1}$ bundle over $\mathcal{M}$, whose connection is given by the $\mathrm{SU}(2)$ part $\vec{p}$ of the Levi-Civita connection on $\mathcal{M}$. We denote by $\pi$ the projection from $\mathcal{Z}$ to $\mathcal{M}$. $\mathcal{Z}$ admits a canonical integrable complex structure and a $\left(\right.$ pseudo $\left.^{22}\right)$ Kähler-Einstein metric, ${ }^{21}$ 


$$
\mathrm{d} s_{\mathcal{Z}}^{2}=\frac{|D t|^{2}}{(1+t \bar{t})^{2}}+\frac{\Lambda}{12} \mathrm{~d} s_{\mathcal{M}}^{2}
$$

Here $t$ is a complex stereographic coordinate on $C P^{1}$ and $D t$ is a one-form of Dolbeault type $(1,0)$ on $\mathcal{Z}$,

$$
D t \equiv \mathrm{d} t+p^{+}-\mathrm{i} p^{3} t+p^{-} t^{2},
$$

where we define $v^{ \pm} \equiv-\frac{1}{2}\left(v^{1} \mp \mathrm{i} v^{2}\right)$ for any real vector $\vec{v}$. Under $\mathrm{SU}(2)$ frame rotations, $\vec{p}$ transforms as a $\mathrm{SU}(2)$ connection while $t$ undergoes Moebius transformations. More concretely, under the infinitesimal transformations,

$$
\delta t=\epsilon^{+}-\mathrm{i} \epsilon^{3} t+\epsilon^{-} t^{2}, \quad \delta p^{+}=-\mathrm{i} \epsilon^{3} p^{+}+\mathrm{i} \epsilon^{+} p^{3}-\mathrm{d} \epsilon^{+}, \quad \delta p^{3}=2 \mathrm{i}\left(\epsilon^{-} p^{+}-\epsilon^{+} p^{-}\right)-\mathrm{d} \epsilon^{3},
$$

$D t$ transforms homogeneously and the first term in (11) is gauge invariant.

While $D t$ is only defined up to a multiplicative factor, its kernel is globally well defined and endows $\mathcal{Z}$ with a canonical complex contact structure. ${ }^{21}$ As explained in Refs. 19 and 23 the contact structure $\mathcal{X}$ descends naturally from the Liouville one-form on the Swann bundle (or in physics parlance, hyper-Kähler cone) $\mathcal{S}$. In practice, it is convenient to use an open covering $\mathcal{U}_{i}$ of $\mathcal{Z}$ and represent the complex contact structure by an holomorphic one-form $\mathcal{X}^{[i]}$ defined on each patch $\mathcal{U}_{i}$, such that $\mathcal{X}^{[i]}$ and $\mathcal{X}^{[j]}$ differ on the overlap $\mathcal{U}_{i} \cap \mathcal{U}_{j}$ by multiplication by a nonvanishing holomorphic function. While the $(1,0)$ form $D t$ is not holomorphic (i.e., $\bar{\partial}$-closed), it lies in the kernel of the complex contact distribution, so on each patch (after suitable refinement) there exists a complex-valued function $\Phi^{[i]}$, such that ${ }^{19,23,24}$

$$
\mathcal{X}^{[i]}=2 \mathrm{e}^{\Phi^{[i]}} \frac{D t}{t} .
$$

The function $\Phi^{[i]}$, which we call the contact potential, is nonholomorphic on $\mathcal{Z}$ but its restriction to each fiber is, so we denote it as $\Phi^{[i]}\left(x^{\mu}, t\right)$, where $x^{\mu}$ are coordinates on $\mathcal{M}$. It is of course subject to the same ambiguity as the contact one-form $\mathcal{X}^{[i]}$, namely,

$$
\left(\mathcal{X}^{[i]}, \Phi^{[i]}\right) \sim\left(f_{i}^{2} \mathcal{X}^{[i]}, \Phi^{[i]}+\log \left(f_{i}^{2}\right)\right),
$$

where $f_{i}^{2}$ is a holomorphic function on $\mathcal{U}_{i}$. Moreover, on the overlap of two different patches, there must exist holomorphic gluing functions $f_{i j}^{2}$, such that

$$
\mathcal{X}^{[i]}=f_{i j}^{2} \mathcal{X}^{[j]}, \quad \Phi^{[i]}-\Phi^{[j]}=\log f_{i j}^{2} .
$$

Combining (8), (14), and (11), it is now obvious that the contact potential determines a Kähler potential on $\mathcal{Z}$ via

$$
K^{[i]}=\log \left(2 \frac{1+t \bar{t}}{|t|}\right)+\operatorname{Re} \Phi^{[i]}\left(x^{\mu}, t\right),
$$

and that ambiguity (15) amounts to Kähler transformations. Moreover, the Swann bundle $\mathcal{S}$, with hype-Kähler metric, ${ }^{17,20}$

$$
\mathrm{d} s_{\mathcal{S}}^{2}=\mathrm{e}^{\lambda^{[i]}+\lambda^{-[i]}+K^{[i]}}\left(\left|\mathrm{d} \lambda^{[i]}+\partial K^{[i]}\right|^{2}+\mathrm{d} s_{\mathcal{Z}}^{2}\right)=|D \pi|^{2}+|\pi|^{2} \mathrm{~d} s_{\mathcal{M}}^{2},
$$

where $\pi^{A^{\prime}}$ parametrizes the $\mathrm{C}^{2} / \mathbb{Z}^{2}$ fiber of $\mathcal{S}, D \pi^{A^{\prime}}=\mathrm{d} \pi^{A^{\prime}}+p^{A^{\prime}}{ }_{B^{\prime}} \pi^{B^{\prime}}$ is the covariant derivative of $\pi^{A^{\prime}}$, and $\mathrm{e}^{\lambda^{[i]}}$ is a local coordinate on the $\mathcal{O}(-1)$ line bundle over $\mathcal{Z}$, can be reconstructed from the contact potential $\Phi^{[i]}$ via 


$$
\pi^{A^{\prime}}=\mathrm{e}^{\lambda^{[i]}-1 / 2 \Phi^{[i]}}\left(\begin{array}{c}
t^{1 / 2} \\
t^{-1 / 2}
\end{array}\right) .
$$

This relation was first obtained in the case of toric quaternion-Kähler manifolds in Ref. 20, but holds, in fact, in general, as can be seen by combining Eqs. 2.14, 20, 22, 77, and 79 in Ref. 19.

\section{B. Darboux coordinates and transition functions}

By Darboux theorem, any contact structure is locally trivial. Thus, possibly after refining the covering, it is possible to choose complex coordinates on $\mathcal{U}_{i}$, such that the complex contact form becomes canonical, i.e.,

$$
\mathcal{X}^{[i]} \equiv \mathrm{d} \alpha^{[i]}+\xi^{[i]} \mathrm{d} \mathcal{\xi}^{i]} .
$$

On the overlap $\mathcal{U}_{i} \cap \mathcal{U}_{j}$, the two coordinate systems must be related by a complex contact transformation. In analogy with classical mechanics, ${ }^{25}$ such transformations may be parametrized via a transition function $H^{[i j]}\left(\xi^{[i]}, \xi^{[j]}, \alpha^{[j]}\right)$ of the "initial position" $\xi^{[i]}$, "final momentum" $\xi^{j j]}$, and "final action" $\alpha^{[j]}$, such that, on $\mathcal{U}_{i} \cap \mathcal{U}_{j}$,

$$
\xi^{[j]}=\xi^{[i]}+T^{[i j]}, \quad \widetilde{\xi}^{[j]}=\tilde{\xi}^{[i]}+\widetilde{T}^{[i j]}, \quad \alpha^{[j]}=\alpha^{[i]}+\widetilde{T}_{\alpha}^{[i j]},
$$

where we defined

$$
\begin{gathered}
T^{[i j]} \equiv-\partial_{\xi \xi j]}\left[H^{[i j]}+\xi^{[j]} \partial_{\alpha}^{[j]} H^{[i j]}\right. \\
\widetilde{T}^{[i j]} \equiv \partial_{\xi^{[i]}} H^{[i j]}, \quad \widetilde{T}_{\alpha}^{[i j]} \equiv H^{[i j]}-\xi^{[i]} \partial_{\xi^{[i]}} H^{[i j]} .
\end{gathered}
$$

The transition functions are subject to consistency conditions on triple overlaps $\mathcal{U}_{i} \cap \mathcal{U}_{j} \cap \mathcal{U}_{k}$, gauge invariance on $\mathcal{U}_{i}$, and reality conditions as described in Refs. 19 and 23. The original quaternion-Kähler metric on $\mathcal{M}$ may be recovered from this holomorphic data by constructing the "real twistor lines," i.e., by solving gluing conditions (21) and expressing the complex coordinates $\xi^{[i]}, \mathcal{E}^{i]}, \alpha^{[i]}$ on $\mathcal{Z}$ in terms of the fiber coordinate $t$ and the base coordinates $x^{\mu}$. The SU(2) connection $\vec{p}$ can then be read off by Taylor expanding $\mathcal{X}^{[i]}$ around any point $t=t_{i}$ in $\mathcal{U}_{i}$ and using (14) and (12).

\section{Integral equations for the twistor lines}

The Darboux coordinates $\xi^{[i]}, \tilde{\xi}^{i}, \alpha^{[i]}$, viewed as functions of the fiber coordinate $t$ for a fixed point $x^{\mu} \in \mathcal{M}$, are usually required to be analytic inside the patch $\mathcal{U}_{i}$. Upon analytic continuation outside $\mathcal{U}_{i}$, one generically encounters singularities. As demonstrated in Refs. 19 and 23 expanding around such a singular point can significantly simplify the retrieval of the metric on $\mathcal{M}$. In particular, it is useful to relax slightly the condition of regularity of the Darboux coordinates and allow for a simple pole in the Darboux coordinate $\xi$ at $t=0$ and at the antipodal point $t=\infty$, while $\widetilde{\xi}$ and $\alpha$ can have a logarithmic singularity at these points. This singularity structure emerges naturally from the superconformal quotient of toric hyper-Kähler manifolds and continues to hold for a class of finite deformations, although we do not expect it to be generally valid. As will become clear in Sec. III, the advantage of such a choice is that the section $t\left(x^{\mu}\right)=0$ is the vanishing locus of a holomorphic function, namely, $1 / \xi=0$, and therefore leads to a (local) integrable complex structure on $\mathcal{M}$.

To keep our notations consistent with Refs. 19 and 23, we assume that $t_{0}=0$ belongs to the "north pole" patch $\mathcal{U}_{+}$. According to our assumption, the Darboux coordinates must therefore behave at $t=0$ as ${ }^{19}$

$$
\xi^{[+]}=\xi_{-1}^{[+]} t^{-1}+\xi_{0}^{[+]}+\xi_{1}^{[+]} t+\mathcal{O}\left(t^{2}\right)
$$




$$
\begin{gathered}
\tilde{\xi}^{[+]}=c_{\tilde{\xi}} \log t+\widetilde{\xi}_{0}^{++]}+\widetilde{\xi}_{1}^{++]} t+\mathcal{O}\left(t^{2}\right), \\
\alpha^{[+]}=c_{\alpha} \log t+c_{\tilde{\xi}} \tilde{\xi}_{-1}^{[+]} t^{-1}+\alpha_{0}^{[+]}+\alpha_{1}^{[+]} t+\mathcal{O}\left(t^{2}\right), \\
\Phi^{[+]}=\phi_{0}^{[+]}+\phi_{1}^{[+]} t+\mathcal{O}\left(t^{2}\right) .
\end{gathered}
$$

The coefficients $c_{\alpha}$ and $c_{\tilde{\xi}}$ of the logarithmic singularity are real numbers sometimes called "anomalous dimensions." Together with the transition functions, they completely specify the twistor space and the original quaternion-Kähler manifold.

Assuming behavior (23) at $t=0$ and enforcing the reality conditions described in Refs. 19 and 23 , gluing conditions (21) can be rewritten as the following exact integral equations, ${ }^{26}$

$$
\begin{gathered}
\xi^{[i]}\left(t, x^{\mu}\right)=A+\mathcal{R}\left(t^{-1}-t\right)+\frac{1}{2} \sum_{j} \oint_{C_{j}} \frac{\mathrm{d} t^{\prime}}{2 \pi \mathrm{i} t^{\prime}} \frac{t^{\prime}+t}{t^{\prime}-t} T^{[+j]}\left(t^{\prime}\right), \\
\widetilde{\xi}^{i i]}\left(t, x^{\mu}\right)=\frac{\mathrm{i}}{2} B+\frac{1}{2} \sum_{j} \oint_{C_{j}} \frac{\mathrm{d} t^{\prime}}{2 \pi \mathrm{i} t^{\prime}} \frac{t^{\prime}+t}{t^{\prime}-t} \widetilde{T}^{++j]}\left(t^{\prime}\right)+c_{\tilde{\xi}} \log t, \\
\alpha^{[i]}\left(t, x^{\mu}\right)=\frac{\mathrm{i}}{2} B_{\alpha}+\frac{1}{2} \sum_{j} \oint_{C_{j}} \frac{\mathrm{d} t^{\prime}}{2 \pi \mathrm{i} t^{\prime}} \frac{t^{\prime}+t}{t^{\prime}-t} \widetilde{T}_{\alpha}^{++j]}\left(t^{\prime}\right)+c_{\alpha} \log t+c_{\tilde{\xi}} \mathcal{R}\left(t^{-1}+t\right),
\end{gathered}
$$

where $C_{j}$ is a contour surrounding the patch $\mathcal{U}_{j}$ and $x^{\mu}=\left\{\mathcal{R}, A, B, B_{\alpha}\right\}$ are real parameters which can be used as coordinates on $\mathcal{M}$. These equations generalize the Riemann-Hilbert problem discussed in Ref. 27 in the context of hyper-Kähler geometry and determine the twistor lines as a formal power series in $H$, which we assume to be convergent.

Having determined the twistor lines in this fashion, the contact potential can obtained as

$$
\Phi^{[i]}\left(t, x^{\mu}\right)=\phi\left(x^{\mu}\right)-\frac{1}{2} \sum_{j} \oint_{C_{j}} \frac{\mathrm{d} t^{\prime}}{2 \pi \mathrm{i} t^{\prime}} \frac{t^{\prime}+t}{t^{\prime}-t} \log \left(1-\partial_{\alpha[j]} H^{[+j]}\left(t^{\prime}\right)\right),
$$

where the real function $\phi\left(x^{\mu}\right)$ (the real part of $\Phi^{[+]}$evaluated at $t=0$ ) is determined in terms of the base coordinates via

$$
\mathrm{e}^{\phi\left(x^{\mu}\right)}=\frac{\mathcal{R}}{4} \sum_{j} \oint_{C_{j}} \frac{\mathrm{d} t^{\prime}}{2 \pi \mathrm{i} t^{\prime}}\left(t^{\prime-1}-t^{\prime}\right) \widetilde{T}^{++j]}\left(t^{\prime}\right)+\frac{1}{2}\left(c_{\tilde{\xi}} A+c_{\alpha}\right) .
$$

Note that (25) reduces to $\Phi^{[i]}\left(t, x^{\mu}\right)=\phi\left(x^{\mu}\right)$ in the special case where the transition functions $H^{[i j]}$ are independent of the action variable $\alpha^{[j]}$. This is the relevant case when $\mathcal{M}$ admits one isometry, as discussed later in Sec. IV.

Following the method outlined in Sec. II B, one may extract the SU(2) connection by expanding around $t=0$,

$$
\begin{aligned}
& p_{+}=\frac{1}{2} \mathrm{e}^{-\phi_{0}^{[+]}}\left(\xi_{-1}^{[+]} \mathrm{d} \widetilde{\xi}_{0}^{\tilde{+}]}+c_{\tilde{\xi}} \mathrm{d} \xi_{-1}^{[+]}\right), \\
& p_{3}=\frac{\mathrm{i}}{2} \mathrm{e}^{-\phi_{0}^{[+]}}\left(\mathrm{d} \alpha_{0}^{[+]}+\xi_{0}^{[+]} \mathrm{d} \widetilde{\xi}_{0}^{++]}+\xi_{-1}^{[+]} \mathrm{d} \widetilde{\xi}_{1}^{[+]}\right)-\mathrm{i} \phi_{[+]}^{1} p_{+},
\end{aligned}
$$

where $\phi_{[+]}^{0}, \phi_{[+]}^{1}$ can be expressed in terms of the Laurent coefficients of the contact twistor lines,

$$
\mathrm{e}^{\phi_{0}^{[+]}}=\frac{1}{2}\left(\xi_{-1}^{[+]} \widetilde{\xi}_{1}^{[+]}+c_{\tilde{\xi}} \tilde{\xi}_{0}^{[+]}+c_{\alpha}\right)
$$




$$
\phi_{1}^{[+]}=\frac{1}{2} \mathrm{e}^{-\phi_{0}^{[+]}}\left(\alpha_{1}^{[+]}+2 \xi_{-1}^{[+]} \widetilde{\xi}_{2}^{++]}+\xi_{0}^{[+]} \widetilde{\xi}_{1}^{++]}+c \widetilde{\xi}^{[+]}\right) .
$$

By expanding the holomorphic one-forms $\mathrm{d} \xi^{[+]}, \mathrm{d} \xi^{++]}$and $\mathrm{d} \alpha$ around $t=0$ and projecting along the base $\mathcal{M}$, one may also obtain a basis of $(1,0)$-forms on $\mathcal{M}$ for the quaternionic structure $J_{3}$. Finally, by using the second equation in (10), one may further compute the quaternionic 2-forms, and finally obtain the quaternion-Kähler metric on $\mathcal{M}$.

\section{Constraints on the Kähler potential}

As explained in Ref. 17, the existence of a complex contact structure implies certain constraints on the Kähler potential $K\left(u^{i}, \bar{u}^{i}\right){ }^{28}$ Let us define the holomorphic two-form $\omega_{i j}$, Reeb vector $\mathcal{Y}^{i}$, and (antisymmetric) bivector $\hat{\omega}^{i j}$ via

$$
\omega_{i j}=-\partial_{[i} \mathcal{X}_{j]}, \quad \mathcal{X}_{i} \mathcal{Y}^{i}=1, \quad \omega_{i j} \mathcal{Y}^{j}=0, \quad \hat{\omega}^{i k} \omega_{k j}=-\delta_{j}^{i}+\mathcal{Y}^{i} \mathcal{X}_{j} .
$$

Then the constraints that $\mathcal{Z}$ is the twistor space of a quaternion-Kähler manifold can be summarized by the three equations,

$$
\begin{gathered}
\mathcal{Y}^{i}=\mathcal{X}^{i} \mathrm{e}^{-2 K}-\hat{\omega}^{i j} K_{j}, \quad \hat{\omega}^{i j}=\left(\omega^{i j}+K^{i} \mathcal{X}^{j}-K^{j} \mathcal{X}^{i}\right) \mathrm{e}^{-2 K}, \\
\operatorname{det} K_{i \bar{j}}=\frac{1}{4} \mathrm{e}^{-4 K},
\end{gathered}
$$

where the indices are raised and lowered with the metric $K_{i j}^{-}$and its inverse $K^{i \bar{j}}$. In particular, the requirement that the metric on $\mathcal{Z}$ should be Kähler-Einstein is Monge-Ampère-Liouville equation (31). Moreover, it follows from (29) that $\hat{\omega}^{i j} \mathcal{X}_{j}=0$, and from (30) that $\mathcal{X}_{i} \mathcal{X}^{i}=\mathrm{e}^{-2 K}$ : the Kähler potential on $\mathcal{Z}$ is thus obtained as the logarithm of the norm of the contact structure.

In Darboux coordinates, the above constraints can be made more explicit. Indeed, conditions (29) uniquely specify

$$
\omega=-\mathrm{d} \xi \wedge \mathrm{d} \tilde{\xi}, \quad \mathcal{Y}=\partial_{\alpha}, \quad \hat{\omega}=2 \xi \partial_{\xi} \wedge \partial_{\alpha}-2 \partial_{\xi} \wedge \partial_{\tilde{\xi}},
$$

while constraints (30) reduce to the following three equations:

$$
\begin{gathered}
K_{\xi}=\frac{1}{2}\left(K^{\tilde{\xi} \bar{\alpha}}+\bar{\xi} K^{\tilde{\xi} \overline{\tilde{\xi}}}\right) \mathrm{e}^{-2 K}, \\
K_{\tilde{\xi}}-\xi K_{\alpha}=-\frac{1}{2}\left(K^{\xi \bar{\xi}}+\bar{\xi} K^{\xi \overline{\tilde{\xi}}}\right) \mathrm{e}^{-2 K}, \\
\mathrm{e}^{2 K}=K^{\alpha \bar{\alpha}}+\xi K^{\tilde{\xi} \bar{\alpha}}+\bar{\xi} K^{\alpha \overline{\tilde{\xi}}}+|\xi|^{2} K^{\tilde{\tilde{\xi} \xi}},
\end{gathered}
$$

to be supplemented by Monge-Ampère equation (31). These constraints will be useful in the next section when deriving master equation (1) from the twistor space.

\section{PRZANOWSKI METRICS FROM TWISTOR SPACE}

Having recalled generalities about quaternion-Kähler spaces in Sec. II, we now come to the main goal of this note, which is to clarify the geometric nature of Przanowski metric (2) and to understand when different solutions of master equation (1) lead to quaternion-Kähler metrics that are related by diffeomorphisms. All of this section holds independent of the assumptions made in Sec. II C, except when we explicitly use some properties of the twistor lines for the $t=0$ slice leading to (56). At the end of this section, we also show how the full twistor space can be recovered from a single "tomogram," or a Przanowski solution, by solving a recursive system of 
differential equations. This system simplifies considerably in the presence of isometries. In particular, the contact potential can be found exactly, see (69), though the twistor lines must still be obtained recursively.

\section{A. From self-dual Einstein spaces to Przanowski metrics}

As already mentioned in Sec. I, metric (2) is manifestly Hermitian with respect to a local integrable complex structure $J$ with complex coordinates $x^{\mu}=\left(z^{1}, z^{2}, \bar{z}^{1}, \bar{z}^{2}\right)$. To show that metric (2) is quaternion-Kähler, it suffices to check that conditions (10) are satisfied for

$$
\begin{gathered}
p^{+}=\mathrm{e}^{h / 2} \mathrm{~d} z^{2}, \quad p^{3}=\frac{i}{2}(\partial-\bar{\partial}) h, \quad p^{-}=\left(p^{+}\right)^{*}, \\
\omega^{+}=\frac{6}{\Lambda} \mathrm{e}^{h / 2} h_{1} \mathrm{~d} z^{1} \wedge \mathrm{d} z^{2}, \quad \omega^{3}=2 \mathrm{i} g_{\alpha \bar{\beta}} \mathrm{d} z^{\alpha} \wedge \mathrm{d} z^{\bar{\beta}}, \quad \omega^{-}=\left(\omega^{+}\right)^{*},
\end{gathered}
$$

where

$$
g_{\alpha \bar{\beta}}=-\frac{3}{\Lambda}\left(\partial_{\alpha} \partial_{\bar{\beta}} h+2 \delta_{\alpha}^{2} \delta_{\bar{\beta}}^{-} \mathrm{e}^{h}\right)
$$

and $\partial=\mathrm{d} z^{1} \partial_{1}+\mathrm{d} z^{2} \partial_{2}$ is the Dolbeault operator associated with $J$. A straightforward computation shows that (10) indeed holds provided $h$ satisfies the master equation (1). Equations (34) and (35) were obtained in a different local SU(2) frame in Ref. 29. One virtue of the above choice is that $\omega_{+}$and $p_{+}$are of Dolbeault type $(2,0)$ and $(1,0)$ with respect to $J$. This would of course also continue to hold after $\mathrm{U}(1)$ frame rotations,

$$
\omega^{+} \rightarrow \mathrm{e}^{\mathrm{i} \theta} \omega^{+}, \quad \omega^{3} \rightarrow \omega^{3}, \quad p^{+} \rightarrow \mathrm{e}^{\mathrm{i} \theta} p^{+}, \quad p^{3} \rightarrow p^{3}+\mathrm{d} \theta
$$

for any real function $\theta\left(x^{\mu}\right)$.

To show that any self-dual Einstein metric can be locally cast into the Przanowski ansatz, we start by choosing a linear combination $J=n_{a}\left(x^{\mu}\right) J^{a} ; a=1,2,3$ of the quaternionic structures, where $\vec{n}$ is a unit three-vector, such that $J$ is (locally) an integrable complex structure. As discussed in Sec. III B, there are infinitely many choices of $n_{a}\left(x^{\mu}\right)$ corresponding to choices of complex submanifolds in the twistor space $\mathcal{Z}$. Upon performing a suitable $\mathrm{SU}(2)$ frame rotation, there is no loss of generality in assuming that $J=J_{3}$. The corresponding quaternionic form $\omega^{+}$and connection $p^{+}$are then of Dolbeault type $(2,0)$ and $(1,0)$ with respect to $J$. One may now perform a suitable holomorphic change of variable such that $p^{+}$is proportional to the holomorphic one-form $\mathrm{d} z^{2}$, i.e.,

$$
p^{+}=\mathrm{e}^{\mathcal{A}} \mathrm{d} z^{2}
$$

for some complex-valued function $\mathcal{A}\left(x^{\mu}\right)$. Clearly, $z^{2}$ is ambiguous up to holomorphic changes of variables of the form (5), whereby $\mathcal{A}$ changes as $\mathcal{A} \rightarrow \mathcal{A}-\log g_{2}\left(z^{2}\right)$. Conditions (10), written in component as

$$
\mathrm{d} \omega^{+}+\mathrm{i} p^{+} \wedge \omega^{3}-\mathrm{i} p^{3} \wedge \omega^{+}=0, \quad \mathrm{~d} \omega^{3}-2 \mathrm{i} p^{+} \wedge \omega^{-}+2 \mathrm{i} p^{-} \wedge \omega^{+}=0
$$

for the curvature two-forms, and

$$
\mathrm{d} p^{+}+\mathrm{i} p^{+} \wedge p^{3}=\frac{\Lambda}{6} \omega^{+}, \quad \mathrm{d} p^{3}-2 \mathrm{i} p^{+} \wedge p^{-}=\frac{\Lambda}{6} \omega^{3}
$$

then uniquely specify 


$$
p^{3}=\mathrm{i}\left(\partial_{\alpha} \overline{\mathcal{A}} \mathrm{d} z^{\alpha}-\partial_{\bar{\alpha}} \mathcal{A} \mathrm{d} \bar{z}^{\alpha}\right), \quad \omega^{+}=\frac{12}{\Lambda} \mathrm{e}^{\mathcal{A}} \partial_{z^{1}}(\operatorname{Re} \mathcal{A}) \mathrm{d} z^{1} \wedge \mathrm{d} z^{2} .
$$

Computing $\omega^{3}$ from the second equation in (39) and multiplying with the complex structure $J^{3}$ then leads to metric (2), where the Przanowski function is identified with the real part of $\mathcal{A}$,

$$
h=\mathcal{A}+\overline{\mathcal{A}}
$$

In particular, under holomorphic changes of variables of the form (5), $h$ changes as prescribed in (6). On the other hand, the imaginary part of $A$ can be shifted away after a U(1) rotation, since $\mathcal{A} \rightarrow \mathcal{A}+\mathrm{i} \theta$ induces rotation (37) on the connection and quaternionic forms. The choice made in Appendix C.2 of Ref. 29 corresponds to $\mathcal{A}-\overline{\mathcal{A}}=\log \left(h_{1}^{-} / h_{1}\right)$, while the one in (34) corresponds to $\mathcal{A}-\overline{\mathcal{A}}=0$.

\section{B. From twistor spaces to Przanowski metrics}

We now discuss how to obtain Przanowski metric (2) in a top-down approach, starting from the twistor space $\mathcal{Z}$. Recall that for any Kähler-Einstein space $\mathcal{Z}$ equipped with a complex contact structure $\mathcal{X}$, the quadratic differential,

$$
\mathrm{d} s_{\mathcal{M}}^{2} \equiv \frac{12}{\Lambda}\left(\mathrm{d} s_{\mathcal{Z}}^{2}-\mathrm{e}^{-2 K}|\mathcal{X}|^{2}\right),
$$

has a two-dimensional kernel and signature $(4,0)$. Its restriction to any real-codimension 2 (local) submanifold $\mathcal{C}$ transverse to the contact distribution, i.e., such that $\left.\mathcal{X}\right|_{\mathcal{C}} \neq 0$, produces local selfdual Einstein metrics on $\mathcal{M}$ that are diffeomorphic to each other. Suppose now that $\mathcal{C}$ is a complex submanifold of $\mathcal{Z}$, given by the vanishing of some holomorphic function $\mathcal{C}\left(u^{i}\right)=0$ on $\mathcal{Z}$. The first term in (43) restricts to a Kähler metric, locally defined on $\mathcal{M}$, whose Kähler potential $K_{\mathcal{C}}$ is the restriction of the Kähler potential $K$ on $\mathcal{Z}$ to the submanifold $\mathcal{C}$. As for the second term, two possibilities arise:

(i) either the two-form $\left.\mathrm{d} \mathcal{X}\right|_{\mathcal{C}}=0$ vanishes on $\mathcal{C}$; in this case there exists a holomorphic function $z^{2}$, such that $\left.\mathcal{X}\right|_{\mathcal{C}}=\mathrm{d} z^{2}$; since $z^{2}$ is nonconstant, it can be taken as a complex coordinate on an open subset in $\mathcal{C}$, and supplemented by a second coordinate $z^{1}$ on $\mathcal{C}$ such that $\mathrm{d} z^{1} \wedge \mathrm{d} z^{2} \neq 0$.

(ii) or the two-form $\left.\mathrm{d} \mathcal{X}\right|_{\mathcal{C}}$ is nondegenerate on $\mathcal{C}$; by Darboux's theorem, there exists complex coordinates $z^{1}, z^{2}$ on an open subset in $\mathcal{C}$ such that $\left.\mathcal{X}\right|_{\mathcal{C}}=z^{1} \mathrm{~d} z^{2}$.

Both cases can be treated simultaneously by assuming that

$$
\left.\mathcal{X}\right|_{\mathcal{C}}=\mathrm{e}^{F_{\mathcal{C}}\left(z^{1}, z^{2}\right)} \mathrm{d} z^{2}
$$

on an open subset of $\mathcal{C}$, coordinatized by complex coordinates $z^{1}, z^{2}$. This covers cases (i) and (ii) above, with $\mathrm{e}^{F_{\mathcal{C}}}=1$ and $\mathrm{e}^{F_{\mathcal{C}}}=z^{1}$, respectively, but allows for more general coordinate choices. Identifying

$$
h=-2 K_{\mathcal{C}}+F_{\mathcal{C}}+\bar{F}_{\mathcal{C}}
$$

we see that metric (43) reduces to the Przanowski form; in particular, $h$ defined in (45) must be a solution of the master equation (1). In Appendix A, we show how this follows from the constrains of twistor geometry discussed in Sec. II D for the special choice $\mathcal{C}\left(u^{i}\right)=\xi$, but the derivation can be easily generalized to any slice. Moreover, quaternionic structures (35) can also be obtained from the twistor space using $^{17}$ 


$$
\omega_{\alpha \bar{\beta}}^{3}=-\mathrm{i}\left(K_{\alpha \bar{\beta}}-\mathrm{e}^{-2 K} \mathcal{X}_{\alpha} \mathcal{X}_{\bar{\beta}}^{-}\right), \quad \omega_{\alpha \beta}^{+}=\mathrm{e}^{-K}\left(\omega_{\alpha \beta}+K_{\alpha} \mathcal{X}_{\beta}-K_{\beta} \mathcal{X}_{\alpha}\right) .
$$

As in (38), Eq. (44) determines $z^{2}$ up to holomorphic changes of variables of the form (5), whereby $F_{\mathcal{C}}$ changes by $F_{\mathcal{C}} \rightarrow F_{\mathcal{C}}-\log g_{2}\left(z^{2}\right)$, reproducing the holomorphic ambiguity of $h$ in (6). More significantly, however, changing the complex submanifold $\mathcal{C}$ produces new solutions of master equation (1) which describe the same metric $\mathcal{M}$ but are related to each other by nonholomorphic changes of variables, as we shall discuss in Sec. III C.

In practice, it is useful to choose Darboux coordinates $u^{i}=(\alpha, \xi, \widetilde{\xi})$ on $\mathcal{Z}$, such that the contact structure $\mathcal{X}$ takes the form (20), and parametrize $\mathcal{M}$ by solving the condition $\mathcal{C}(u)=0$ for one of the coordinates $u^{i}$ in terms of the other. In the remainder of this subsection, we illustrate this process for several choices of parametrizations.

\section{1. $\hat{\xi}$-parametrization}

Suppose the submanifold $\mathcal{C}$ can be described locally as $\xi=\hat{\xi}(\alpha, \widetilde{\xi})$, where $\hat{\xi}$ is any holomorphic function of $\alpha, \tilde{\xi}$. Condition (44) then reduces to the three conditions,

$$
\partial z^{2}=\mathrm{e}^{-F} \hat{\xi} \hat{\xi}, \quad \partial_{\alpha} z^{2}=\mathrm{e}^{-F \hat{\xi}}, \quad\left(\partial_{\hat{\xi}}-\hat{\xi} \partial_{\alpha}\right) F_{\hat{\xi}}+\partial_{\alpha} \hat{\xi}=0
$$

for the functions $F_{\hat{\xi}}(\alpha, \widetilde{\xi})$ and $z^{2}(\alpha, \widetilde{\xi})$. The last condition is, in fact, the integrability condition for the first and second. $z^{1}(\alpha, \widetilde{\xi})$ can be chosen arbitrarily in such a way that the Jacobian $\partial\left(z^{1}, z^{2}\right) / \partial(\alpha, \widetilde{\xi})$ is nonzero. Different solutions will be related by holomorphic changes of variables of the form (5).

A particular class of submanifolds $\xi=\hat{\xi}(\alpha, \widetilde{\xi})$ where (47) can be solved explicitly are those of the form $\xi=\partial_{\tilde{\xi}} W(\widetilde{\xi})$, where $W$ is an arbitrary holomorphic function of $\widetilde{\xi}$ : in this case, one may choose

$$
z^{1}=\widetilde{\xi}, \quad z^{2}=\alpha+W(\widetilde{\xi}), \quad F_{\hat{\xi}}=0,
$$

leading to the following infinite class of solutions of Przanowski equation:

$$
h_{W}\left(z^{1}, z^{2}\right)=-2 K\left(W^{\prime}\left(z^{1}\right), z^{1}, z^{2}-W\left(z^{1}\right)\right),
$$

where $W^{\prime}$ denotes the derivative of $W$. The case $W=0$ allows for a particularly simple derivation of the master equation, as outlined in Appendix A.

\section{2. $\hat{\alpha}$-parametrization}

Suppose now that the submanifold $\mathcal{C}$ is described locally as $\alpha=\hat{\alpha}(\xi, \widetilde{\xi})$. Condition (44) now reduces to the three conditions,

$$
\begin{gathered}
\partial_{\xi} z^{2}=\mathrm{e}^{-F_{\hat{\alpha}}} \partial_{\xi} \hat{\alpha}, \quad \partial_{\tilde{\xi}} z^{2}=\mathrm{e}^{-F_{\hat{\alpha}}}\left(\xi+\partial_{\tilde{\xi}} \hat{\alpha}\right) . \\
\partial_{\xi} \hat{\alpha} \partial_{\tilde{\xi}} F_{\hat{\alpha}}-\partial_{\tilde{\xi}} \hat{\alpha} \partial_{\xi} F_{\hat{\alpha}}=\xi \partial_{\xi} F_{\hat{\alpha}}-1 .
\end{gathered}
$$

Again, the third equation is the integrability condition for the first two.

\section{3. $t=0$ slice}

A particular convenient choice of submanifold $\mathcal{C}$ is the special locus $t\left(x^{\mu}\right)=0$, introduced in Sec. II C, where one of the Darboux coordinates $\xi$ diverges linearly. This may be viewed as a special case of the $\hat{\xi}$-parametrization, given formally by $\hat{\xi}=\infty$. This complex submanifold was used 
in the superconformal quotient constructions of Refs. 17, 30, and 31 and is particularly convenient for reconstructing the twistor space from the Przanowski function $h$ in the presence of one isometry, as will become apparent in Sec. III D.

Since the contact potential $\Phi$ is assumed to be regular on the section $t=0$, Kähler potential (17) diverges at that point. This is repaired by a Kähler transformation,

$$
K \mapsto K^{\prime}=K-\log |\xi|=\operatorname{Re} \Phi-\log \frac{|t \xi|}{2(1+t \bar{t})},
$$

combined with a rescaling of the contact form (20),

$$
\mathcal{X} \mapsto \mathcal{X}^{\prime}=\xi^{-1} \mathcal{X}=\mathrm{d} \widetilde{\xi}+\xi^{-1} \mathrm{~d} \alpha .
$$

Now the limit $t=0$ is regular and yields

$$
K_{t=0}^{\prime}=\phi\left(x^{\mu}\right)-\log \frac{\left|\tilde{\xi}_{-1}^{[+]}\right|}{2},\left.\quad \mathcal{X}^{\prime}\right|_{t=0}=\mathrm{d}\left(\tilde{\xi}_{0}^{[+]}+c \tilde{\xi} \log \tilde{\xi}_{-1}^{[+]}\right),
$$

where $\xi_{-1}^{[+]}, \widetilde{\xi}_{0}^{++]}$are the Laurent coefficients appearing in (23). Defining the complex coordinates $z^{1}, z^{2}$ as

$$
z^{1}=\alpha_{0}^{[+]}+c_{\alpha} \log \xi_{-1}^{[+]}-c \tilde{\xi} A, \quad z^{2}=\widetilde{\xi}_{0}^{[+]}+c_{\tilde{\xi}} \log \xi_{-1}^{[+]},
$$

such that $F_{t=0}=0$, we can finally cast the metric into the Przanowski ansatz with

$$
h=-2 K_{t=0}^{\prime}=-2 \phi\left(x^{\mu}\right)+2 \log \frac{\mathcal{R}}{2} .
$$

\section{Relating different parametrizations}

While each of the parametrizations above have their own virtues, it turns out that they can all be mapped to each other (locally) by a suitable contact transformation. In particular, the slice $\xi$ $=\hat{\xi}(\widetilde{\xi}, \alpha)$ can be mapped to $\xi^{\prime}=\infty$ via the contact transformation,

$$
\begin{gathered}
\xi^{\prime}=-\mathrm{e}^{F} \hat{\xi} /(\xi-\hat{\xi}), \\
\widetilde{\xi}^{\prime}+c_{\tilde{\xi}} \log \xi^{\prime}=z^{2}(\widetilde{\xi}, \alpha)+c_{\alpha}(\xi-\hat{\xi}) \mathrm{e}^{-F \hat{\xi}}, \\
\alpha^{\prime}-c_{\tilde{\xi}} \xi^{\prime}+c_{\alpha} \log \xi^{\prime}=-\widetilde{\xi},
\end{gathered}
$$

where $F_{\xi}$ satisfies differential equation (47). Indeed, it is readily checked that $\mathcal{X}^{\prime} / \xi^{\prime}=\mathrm{e}^{-F_{\hat{\xi}}} \mathcal{X}$.

Similarly, the slice $\alpha=\hat{\alpha}(\xi, \widetilde{\xi})$ can be mapped to the slice $\xi^{\prime}=\infty$ by the following complex contact transformation:

$$
\begin{gathered}
\xi^{\prime}=1 /(\alpha-\hat{\alpha}), \\
\widetilde{\xi}^{\prime}+c_{\tilde{\xi}} \log \xi^{\prime}=z^{2}(\xi, \widetilde{\xi})+\left(\mathrm{e}^{-F \hat{\alpha}}-c_{\alpha}\right)(\alpha-\hat{\alpha}), \\
\alpha^{\prime}-c_{\tilde{\xi}} \xi^{\prime}+c_{\alpha} \log \xi^{\prime}=-\mathrm{e}^{-F_{\hat{\alpha}}},
\end{gathered}
$$

such that $\mathcal{X}^{\prime} / \xi^{\prime}=\mathrm{e}^{-F_{\hat{\alpha}}} \mathcal{X}$. Here $F_{\hat{\alpha}}$ is the function defined by differential equation (51). By combining these two transformations, one can dispose of the singular slice $\xi^{\prime}=\infty$ and relate the $\hat{\xi}$ and $\hat{\alpha}$ parametrizations directly, 


$$
\begin{gathered}
\alpha-\hat{\alpha}=-\mathrm{e}^{-F_{\hat{\xi}^{\prime}}}\left(\xi^{\prime}-\hat{\xi}^{\prime}\right), \\
z^{2}(\xi, \widetilde{\xi})+\mathrm{e}^{-F \hat{\alpha}}(\alpha-\hat{\alpha})=z^{\prime 2}\left(\widetilde{\xi}^{\prime}, \alpha^{\prime}\right), \\
\mathrm{e}^{-F_{\hat{\alpha}}}=\widetilde{\xi}^{\prime},
\end{gathered}
$$

such that $\mathrm{e}^{-F_{\hat{\alpha}}} \mathcal{X}=\mathrm{e}^{-F \hat{\xi}^{\prime}} \mathcal{X}^{\prime}$

\section{Isometric solutions and nonholomorphic coordinate changes}

As indicated previously, different choices of submanifold $\mathcal{C}$ lead to different Przanowski representations of the same metric, where different (local) integrable complex structures are manifest. There is, however, a clear way to relate different representations, depicted in Fig. 1 on p. 2: let

$$
\begin{gathered}
i:\left(z^{1}, z^{2}\right) \in V \rightarrow \mathcal{C} \subset \mathcal{Z}, \\
i^{\prime}:\left(z^{\prime 1}, z^{\prime 2}\right) \in V \rightarrow \mathcal{C}^{\prime} \subset \mathcal{Z}
\end{gathered}
$$

be the two submersions from open subsets $V, V^{\prime}$ in $\mathbb{C}^{2}$ into the submanifolds $\mathcal{C}, \mathcal{C}^{\prime}$, corresponding to two different Przanowski representations. Consider now

$$
z \in V \mapsto\left(i^{\prime}\right)^{-1}\left[\pi^{-1}\left[\pi(i(z)] \cup \mathcal{C}^{\prime}\right] \in V^{\prime},\right.
$$

where $\pi$ is the projection from $\mathcal{Z}$ to $\mathcal{M}$. In writing (61), we have restricted the open subsets $V, V^{\prime}$, such that the twistor line above the point $\pi(i(z))$ intersects $\mathcal{C}^{\prime}$ in one and exactly one point. Equation (61) provides the map between the two coordinate systems $\left(z^{1}, z^{2}\right)$ and $\left(z^{\prime 1}, z^{\prime 2}\right)$. Because the projection $\pi$ is nonholomorphic, this map is also nonholomorphic. Of course, if $i(z)$ belongs to intersection $\mathcal{C} \cap \mathcal{C}^{\prime}$, the map reduces to $\left(i^{\prime}\right)^{-1} \circ i$, so becomes holomorphic, but this generically happens only on a real-codimension 2 subspace of $V, V^{\prime}$.

To illustrate this prescription, we now determine the change of variables between the "special slice" $t=0$, corresponding to $\xi=\infty$, and a nearby slice parameterized by

$$
\hat{\xi}^{-1}=\partial_{\alpha} W(\tilde{\xi}, \alpha),
$$

where $W$ is considered to be infinitesimal. For simplicity, we assume that the anomalous dimensions vanish. In this case it is possible to solve Eqs. (47) to first order in $W$ and obtain

$$
\mathrm{e}^{F \hat{\xi}} \approx \frac{1-\partial_{\tilde{\xi}} W}{\partial_{\alpha} W}, \quad z^{1} \approx \alpha, \quad z^{2} \approx \widetilde{\xi}+W
$$

For $W=0$, the coordinates $z^{\alpha}$ and Przanowski function are given by

$$
z_{(0)}^{1}=\alpha_{0}^{[+]}, \quad z_{(0)}^{2}=\widetilde{\xi}_{0}^{-+]}, \quad h_{(0)}=-2 \phi+2 \log \frac{\mathcal{R}}{2} .
$$

For $W$ nonzero but vanishingly small, perturbing around this solution leads to

$$
\begin{gathered}
z^{1} \approx z_{(0)}^{1}+\mathcal{R} \alpha_{1}^{[+]} \partial_{\alpha} W, \\
z^{2} \approx z_{(0)}^{2}+2 \mathrm{e}^{\phi_{0}^{[+]}} \partial_{\alpha} W+W,
\end{gathered}
$$




$$
h \approx h_{(0)}-2 \operatorname{Re}\left[\partial_{\bar{\xi}} W+\mathcal{R} \phi_{1}^{[+]} \partial_{\alpha} W\right],
$$

which is manifestly nonholomorphic. In particular, the variation

$$
\delta h=h-h_{(0)}-2 \operatorname{Re}\left(h_{1}\left(z^{1}-z_{(0)}^{1}\right)+h_{2}\left(z^{2}-z_{(0)}^{2}\right)\right)
$$

provides an eigenmode of linearized master equation (3) outside the class of trivial eigenmodes (7). Unfortunately, it does not seem possible to express $\delta h$ in terms of $h, W$ and their derivatives only.

\section{Lifting back to the twistor space}

In this section we address the inverse problem: given a solution $h$ of the Przanowski equation, construct the corresponding twistor space, in particular, provide the contact potential and local Darboux coordinates. These are of course defined up to local complex contact transformations (see Sec. II B). Since all local complex submanifolds are equivalent up to a contact transformation, as explained in Sec. III B 4, we can assume that $h$ is associated with the complex submanifold $t\left(x^{\mu}\right)=0$, along the lines of Sec. III B 3 .

Under this assumption, we can address this problem as follows: Eq. (14) relates the Darboux coordinates to the SU(2) connection, which was evaluated in terms of the function $h$ in (38) and (41). The Przanowski coordinates $\left(z^{1}, z^{2}\right)$ can in turn be related to the Darboux coordinates using (55), where the coefficient $\xi_{-1}^{[+]}$is, in general, complex. Moreover, one can use (56) in the form $\phi=-h / 2+\log \left|\xi_{-1}^{[+]}\right| / 2$. Comparing the resulting SU(2) connection with (27) and using (28), the first coefficients in the Laurent expansion of $\xi$ at $t=0$ are found to be

$$
\xi_{-1}^{[+]}=\frac{\mathrm{e}^{h / 2+\mathrm{i} \theta}}{h_{1}}, \quad \xi_{0}^{[+]}=\xi_{-1}^{[+]} \phi_{1}^{[+]}+\frac{h_{2}}{h_{1}},
$$

while the constant part of the contact potential is

$$
\phi_{0}^{[+]}=-\log \left(2 h_{1}\right)
$$

Recall that $\theta=\operatorname{Im} \mathcal{A}$ can be shifted arbitrarily by rotating the complex structures. By choosing $\theta$ $=-(\mathrm{i} / 2) \log \left(h_{1} / h_{1}^{-}\right)$, we can ensure that the coefficient $\xi_{-1}^{[+]}=\mathcal{R}$ is real, as is necessary for (24) to hold.

To determine the higher order Laurent coefficients, one should solve a recursive system of differential equations following from (14). We demonstrate explicitly this in Appendix B in the case when $\mathcal{M}$ admits two commuting Killing vectors. Such geometries can be described by Przanowski solutions $h$ that depend only on $\operatorname{Re} z^{\alpha}$. In this case, the Killing vector $\kappa=\mathrm{i}\left(\partial_{1}-\partial_{1}^{-}\right)$lifts to a holomorphic Killing vector $\kappa_{\mathcal{Z}}=\mathrm{i} \partial_{\alpha}$, and therefore the transition functions $H^{[i j]}$ must not depend on $\alpha$. As explained in Ref. 19, this implies that the contact potential $\Phi^{[i]}$ is the same in all patches and equal to the real function $\phi\left(x^{\mu}\right)$ so that from (68),

$$
\Phi^{[i]}=\phi=-\log \left(2 h_{1}\right) .
$$

On the other hand, the $\mathcal{O}(2)$-valued moment map for the vector field $\kappa^{\prime}=\mathrm{i}\left(\partial_{2}-\partial_{2}\right)$ can be chosen to be one of the complex Darboux coordinates $\xi$, given globally by

$$
\xi=A+\mathcal{R}\left(t^{-1}-t\right)
$$

where $A=\xi_{0}^{[+]}$and $\mathcal{R}$ are real and follow from (67) with vanishing $\theta$, 


$$
\mathcal{R}=\frac{\mathrm{e}^{h / 2}}{h_{1}}, \quad \xi_{0}^{[+]}=\frac{h_{2}}{h_{1}} .
$$

Note that these identifications are valid even in the presence of only one isometry. The remaining complex Darboux coordinates can be found order by order as expansions in $t$ by solving differential equations (B6).

\section{SELF-DUAL EINSTEIN SPACES WITH ISOMETRIES}

We now turn to self-dual Einstein spaces with isometries. In Sec. IV A, we review the relation between self-dual Einstein spaces with one isometry and solutions of the Toda equation and map them to a particular class of solutions of the Przanowski equation, following Ref. 15 with a twist. In Sec. IV B, we consider the case of two commuting isometries, and we show how the Calderbank-Pedersen metrics can be brought into the Toda-form.

\section{A. Canonical complex structure and Toda equation}

As shown by Tod, ${ }^{32}$ self-dual Einstein metrics with one Killing vector field can be written in local coordinates $(\rho, z, \bar{z}, \psi)$ in the form

$$
\mathrm{d} s_{\mathcal{M}}^{2}=-\frac{3}{\Lambda}\left[\frac{P}{\rho^{2}}\left(\mathrm{~d} \rho^{2}+4 \mathrm{e}^{T} \mathrm{~d} z \mathrm{~d} \bar{z}\right)+\frac{1}{P \rho^{2}}(\mathrm{~d} \psi+\Theta)^{2}\right],
$$

where the isometry acts as a shift in the coordinate $\psi$. Here, $T$ is a function of $(\rho, z, \bar{z}), P \equiv 1$ $-\frac{1}{2} \rho \partial_{\rho} T$, and $\Theta$ is a one-form such that

$$
\mathrm{d} \Theta=\mathrm{i}\left(\partial_{z} P \mathrm{~d} z-\partial_{\bar{z}} P \mathrm{~d} \bar{z}\right) \wedge \mathrm{d} \rho-2 \mathrm{i} \partial_{\rho}\left(P \mathrm{e}^{T}\right) \mathrm{d} z \wedge \mathrm{d} \bar{z} .
$$

The integrability condition for (73) follows from the three-dimensional continuous Toda equation,

$$
\partial_{z} \partial_{\bar{z}} T+\partial_{\rho}^{2} \mathrm{e}^{T}=0
$$

which is the requirement coming from the Einstein self-duality condition of the metric. ${ }^{32}$ To see the connection with the more familiar Toda equation from integrable systems, note that (74) follows from the equation

$$
\partial_{z} \partial_{\bar{z}} \mathcal{F}+\mathrm{e}^{\partial_{\rho}^{2} \mathcal{F}}=0, \quad T=\partial_{\rho}^{2} \mathcal{F},
$$

by differentiating twice with respect to $\rho$. Equation (75) is recognized as the continuum limit of the $s l(n)$ Toda system for $n \rightarrow \infty$ and is amenable to the usual integrable techniques, e.g., its solutions can be constructed using free fermions. ${ }^{33-35}$

As stressed already several times, there are infinitely many ways to cast (72) into Przanowski form (2), corresponding to different choices of integrable complex structures on $\mathcal{M}$. However, as explained in Ref. 15, there is one canonical choice of complex structure which makes the Killing vector field particular simple in Przanowski's variables. To specify this complex structure, recall that to any Killing vector field $\kappa$, one may associate the vector-valued moment map $\vec{\mu}$ via $^{36}$

$$
\vec{\mu}_{\kappa}=\frac{1}{2}\left(\vec{r}_{\kappa}+\kappa \cdot \vec{p}\right),
$$

where $\vec{p}$ is the $\mathrm{SU}(2)$ connection and $\vec{r}$ is a three-vector which generates the rotation of the quaternionic two-forms under the Lie-derivative along $\kappa$,

$$
\mathcal{L}_{\kappa} \vec{\omega}+\vec{r}_{\kappa} \times \vec{\omega}=0 .
$$

The moment map $\vec{\mu}$ determines a global holomorphic section of $H^{0}(\mathcal{Z}, \mathcal{O}(2))$, represented in the patch $\mathcal{U}_{i}$ by the holomorphic section, 


$$
\mu_{\kappa}^{[i]} \equiv \mathrm{e}^{\Phi_{[i]}}\left(\mu_{\kappa}^{+} t^{-1}-\mathrm{i} \mu_{\kappa}^{3}+\mu_{\kappa}^{-} t\right) .
$$

In particular, $\mu_{[i]}$ encodes the holomorphic action of $\kappa$ on $\mathcal{Z}$ via $\kappa_{\mathcal{Z}} \cdot \mathcal{X}^{[i]}=\mu_{\kappa}^{[i]}$. Now, the vanishing of $\mu_{[i]}$ determines two sections $t_{ \pm}\left(x^{\mu}\right)$, related by the antipodal map, and therefore one integrable complex structure (and its complex conjugate) canonically associated with the Killing vector $\kappa$. Choosing this complex structure ${ }^{15}$ leads to a Przanowski representation for metric (72) with a "type 1" isometry, i.e., such that $h_{1}=h_{1}^{-37}$ The relation between $h, z^{1}, z^{2}$ and $T, \rho, z$ is given by the Lie-Bäcklund transformation,

$$
z=z^{2}, \quad \rho=1 /\left(2 h_{1}\right), \quad T=h+2 \log \rho .
$$

The moment map for the Killing vector $\kappa_{1}=\mathrm{i}\left(\partial_{z^{1}}-\partial_{\bar{z}^{1}}\right)$ is easily computed to be

$$
\vec{\mu}_{\kappa_{1}}=-\frac{3}{\Lambda}\left(0,0, h_{1}\right),
$$

so that the two sections are $t_{+}\left(x^{\mu}\right)=0$ and $t_{-}\left(x^{\mu}\right)=\infty$. Thus, the complex submanifold $t=0$ plays once again a distinguished rôle, as it produces a Przanowski solution adapted to the Killing field $\kappa$. Using identifications (71) and (69) valid for this slice, the Toda variables $\rho$ and $T$ can therefore be related to the twistorial data,

$$
\rho=\mathrm{e}^{\phi}, \quad T=2 \log (\mathcal{R} / 2) .
$$

For a given Przanowski metric, all isometries are not necessarily of this type, however. Another possibility, but by no means the only one, is if $h_{2}=h_{2}^{-}$, i.e., if $h$ depends only on $z^{2}+\bar{z}^{2}$ : metric (2) now admits a "type 2" Killing field $\kappa_{2}=\mathrm{i}\left(\partial_{z^{2}}-\partial_{\bar{z}^{2}}\right)$, with moment map,

$$
\vec{\mu}_{\kappa_{2}}=-\frac{3}{\Lambda}\left(\mathrm{ie}^{h / 2},-\mathrm{ie}^{h / 2}, h_{2}\right)
$$

corresponding to $t_{+}\left(x^{\mu}\right)=-\frac{1}{2} \mathrm{e}^{h}\left(h_{2} \pm \sqrt{h_{2}^{2}+4 \mathrm{e}^{2 h}}\right)$. It is not known how to relate such solutions of master equation (1) explicitly, but on general ground there must exist a nonholomorphic change of variables which converts the metric into Przanowski's form with a type 1 isometry.

If, however, one knows the twistor space description, such a relation can easily be found. For example, if the isometry is realized on the twistor space as shifts of the complex coordinate $\alpha$, one obtains Przanowski's descriptions with "type 1" or "type 2" isometries by taking the slices $\xi=\infty$ or $\xi=0$, respectively. This follows immediately from the definition of the coordinates $z^{\alpha}$ in (55) and (48). Then the knowledge of explicit expressions for these coordinates allows to find the corresponding nonholomorphic coordinate change.

\section{B. Calderbank-Pedersen meet Toda-Przanowski}

Self-dual Einstein spaces $\mathcal{M}$ with two commuting Killing vectors are described by the Calderbank-Pedersen ansatz, ${ }^{38}$

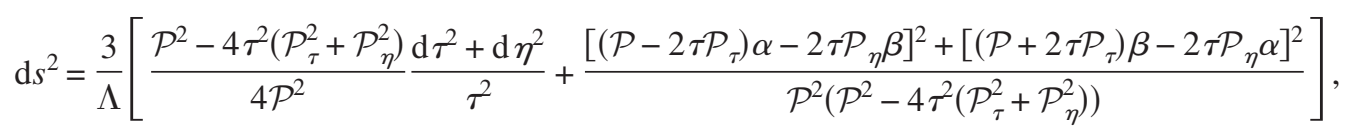

where $x^{\mu}=\{\tau, \eta, \varphi, \psi\}$ are real coordinates on $\mathcal{M}, \alpha=\sqrt{\tau} \mathrm{d} \varphi$, and $\beta=(\eta \mathrm{d} \varphi-\mathrm{d} \psi) / \sqrt{\tau}$ are invariant one-forms under the torus action, and $\mathcal{P}(\tau, \eta)$ is a function satisfying the Laplace equation on the hyperbolic 2-plane with a specific eigenvalue,

$$
\tau^{2}\left(\partial_{\tau}^{2}+\partial_{\eta}^{2}\right) \mathcal{P}(\eta, \tau)=\frac{3}{4} \mathcal{P}(\eta, \tau)
$$

As usual, $\mathcal{P}_{\tau} \equiv \partial_{\tau} \mathcal{P}, \mathcal{P}_{\eta} \equiv \partial_{\eta} \mathcal{P}$, etc. 
We now single out the Killing vector $\partial_{\psi}$ to cast the metric in Toda's form. For this purpose, define $G=\sqrt{\tau} \mathcal{P}$. The metric can be rewritten as ${ }^{39}$

$$
\mathrm{d} s^{2}=-\frac{3}{\Lambda}\left[\frac{P}{G^{2}}\left(\tau^{2} \mathrm{~d} \varphi^{2}+\left(G_{\tau}^{2}+G_{\eta}^{2}\right)\left(\mathrm{d} \tau^{2}+\mathrm{d} \eta^{2}\right)\right)+\frac{1}{P G^{2}}(\mathrm{~d} \psi+\Theta)^{2}\right],
$$

where

$$
P=\frac{\tau\left(G_{\tau}^{2}+G_{\eta}^{2}\right)-G G_{\tau}}{\tau\left(G_{\tau}^{2}+G_{\eta}^{2}\right)}, \quad \Theta=\left(\frac{G G_{\eta}}{G_{\tau}^{2}+G_{\eta}^{2}}-\eta\right) \mathrm{d} \varphi .
$$

Now, introduce new coordinates $z=\frac{1}{2}(V+\mathrm{i} \varphi), \rho=G$, where $V$ is defined, up to an additive constant, by $^{38}$

$$
G_{\tau}=-\tau V_{\eta}, \quad G_{\eta}=\tau V_{\tau} .
$$

The integrability condition for these two equations is $G_{\tau \tau}+G_{\eta \eta}-G_{\tau} / \tau=0$, which follows from (84). Then it is straightforward to check that

$$
\tau^{2} \mathrm{~d} \varphi^{2}+\left(G_{\tau}^{2}+G_{\eta}^{2}\right)\left(\mathrm{d} \tau^{2}+\mathrm{d} \eta^{2}\right)=\mathrm{d} \rho^{2}+4 \mathrm{e}^{T} \mathrm{~d} z \mathrm{~d} \bar{z},
$$

where $T \equiv 2 \log \tau$ can be viewed as a function of $z, \bar{z}, \rho$ (the additive constant ambiguity in $V$ just amounts to an overall translation of the solution in the $z$ plane). Hence (85) is of the form (72), and $T$ satisfies Toda equation (74). Since $T$ is independent of $\psi$, the Przanowski function $h$ defined by (79) is independent of $z^{2}-\bar{z}^{2}$. Therefore, the commuting isometries $\partial_{\psi}$ and $\partial_{\varphi}$ correspond to type 1 and type 2 invariances of the Przanowski function, respectively.

The relation to the Przanowski data and the variables of the twistor formulation can be found from (79) and (81),

$$
\tau=\frac{\mathrm{e}^{h / 2}}{2 h_{1}}=\frac{\mathcal{R}}{2}, \quad \mathcal{P}=\frac{\mathrm{e}^{-h / 4}}{\sqrt{2 h_{1}}}=\mathrm{e}^{\phi} \sqrt{\frac{2}{\mathcal{R}}} .
$$

\section{EXAMPLES}

\section{A. $S^{4}$ and $H^{4}$}

In this subsection we discuss the quaternionic projective space $H P^{1}$ (the four-sphere $S^{4}$ ) and its noncompact analog, the hyperbolic plane $H^{4}$. The hyper-Kähler cones are $\mathbb{H}^{2}$ and $\mathbb{H}^{1,1}$, respectively. The twistor space above the quaternion-Kähler $(\mathrm{QK})$ space is obtained by projectivizing the hyperkahler cone (HKC) with respect to multiplication by complex numbers, so $\mathcal{Z}=\mathrm{C} P^{3}$ or its noncompact version. This space can be covered by four patches, which we can consider separately to get a global construction of the four-dimensional QK space. The data we need to provide to specify the twistor space $\mathcal{Z}$ are the Kähler potential $K_{\mathcal{Z}}$ and the contact form $\mathcal{X}$, on each of the patches. Below we give explicit formulas for one patch, the extension to other patches is relatively straightforward.

As in Ref. 40, we give two equivalent descriptions, which both have their own virtues. The first description is the simplest and most commonly used, but does not obey the scaling properties that are used in the superconformal description of the hyper-Kähler cone. Therefore, many of the properties stated in Sec. II C are not satisfied. We then show how this can be cured in an improved version that we discuss at the end of this subsection. The two versions are related by a simple contact transformation.

\section{First version}

In the first description, we choose local coordinates $u_{1}, u_{2}, u_{3}$ on $\mathcal{Z}=\mathrm{C} P^{3}$ and construct the Fubini-Study metric with Kähler potential, 


$$
K=\log \frac{1}{2}\left(1+\left|u_{1}\right|^{2}+\epsilon\left|u_{2}\right|^{2}+\epsilon\left|u_{3}\right|^{2}\right) .
$$

Here, $\epsilon=1$ for $S^{4}$, and $\epsilon=-1$ for $H^{4}$. Their twistor spaces are Riemannian or pseudo-Riemannian, respectively. The holomorphic contact form is independent of the signature and can locally be written as

$$
\mathcal{X}=\frac{1}{2}\left(\mathrm{~d} u_{1}+u_{2} \mathrm{~d} u_{3}-u_{3} \mathrm{~d} u_{2}\right) .
$$

One can rewrite the contact form to bring it in Darboux form, by changing variables

$$
\alpha=\frac{1}{2}\left(u_{1}-u_{2} u_{3}\right), \quad \xi=u_{2}, \quad \tilde{\xi}=u_{3}, \quad \mathcal{X}=\mathrm{d} \alpha+\xi \mathrm{d} \tilde{\xi} .
$$

Twistor lines can be constructed as

$$
u_{1}=t, \quad u_{2}=z-\epsilon \bar{w} t, \quad u_{3}=w+\epsilon \bar{z} t .
$$

Notice that they are not of the form (24), as explained in the beginning of this subsection.

The boundary of this patch is when the coordinates $u_{1}, u_{2}, u_{3} \rightarrow \infty$ or in terms of the twistor lines, at $t=\infty$, that is, the southpole on the two-sphere. Therefore, the chosen patch on the twistor space projects to a patch on the twistor sphere which excludes the southpole. Moreover, for $\epsilon=$ -1 , there is an additional restriction. Kähler potential (90) implies that we must restrict ourselves to the region,

$$
1+\left|u_{1}\right|^{2}>\left|u_{2}\right|^{2}+\left|u_{3}\right|^{2} \Rightarrow|z|^{2}+|w|^{2}<1 .
$$

This is precisely the defining equation for a unit four-ball $B^{4}$. The four-ball is known to be a stereographic projection of $H^{4}$, so $z$ and $w$ form a coordinate system on the quaternionic projective space.

The contact potential can easily be computed from (14). We find

$$
\mathrm{e}^{\Phi}=\frac{t}{4}\left(1+\epsilon\left(|z|^{2}+|w|^{2}\right)\right)
$$

Note that it is $t$ dependent. Moreover, substituting the twistor lines into the contact form, we get the $\mathrm{SU}(2)$ connection,

$$
p^{+}=\frac{z \mathrm{~d} w-w \mathrm{~d} z}{1+\epsilon\left(|z|^{2}+|w|^{2}\right)}, \quad p^{3}=\mathrm{i} \epsilon \frac{z \mathrm{~d} \bar{z}+w \mathrm{~d} \bar{w}-\bar{z} \mathrm{~d} z-\bar{w} \mathrm{~d} w}{1+\epsilon\left(|z|^{2}+|w|^{2}\right)} .
$$

To determine the Przanowski function, we bring $p^{+}$to the form (38) and follow the procedure outlined there. The easiest choice is to define the Przanowski coordinates as ${ }^{41}$

$$
z^{1}=w, \quad z^{2}=-\frac{z}{w}
$$

such that

$$
h_{\mathcal{C}}=2 \log \left[\frac{\left|z^{1}\right|^{2}}{1+\epsilon\left|z^{1}\right|^{2}\left(1+\left|z^{2}\right|^{2}\right)}\right] .
$$

In terms of slices, this solution can be obtained by taking the special slice $t=0$. Indeed, it corresponds to the slice $\mathcal{C}(u) \equiv u_{1}=0$, and we reproduce (98) from (45) by noting that $F_{\mathcal{C}}=\log \frac{1}{2}\left(z^{1}\right)^{2}$. Changing $z^{1} \rightarrow \mathrm{e}^{z^{1}}$ and dualizing with respect to the type 1 isometry, (98) produces the following solution to Toda equation (74), 


$$
T_{\mathcal{C}}=2 \log \left(\frac{\epsilon}{4} \frac{4 \rho-1}{1+z \bar{z}}\right) .
$$

Taking other slices yields different solutions of the Przanowski equation. For instance, taking $\mathcal{C}^{\prime}(u) \equiv u_{2}=0$, one may choose $z^{\prime 2}=u_{1}, z^{\prime 1}=u_{3}$ and obtain from (45), with $F_{\mathcal{C}^{\prime}}=-\log 2$,

$$
h_{\mathcal{C}^{\prime}}=-2 \log \left(1+\epsilon\left|z^{\prime 1}\right|^{2}+\left|z^{\prime 2}\right|^{2}\right) .
$$

Dualizing this into the Toda form leads back to (99) with $\rho \rightarrow-\rho$, which is obviously still a solution to (74). By comparing the twistor lines on the intersection of the two slices, $t=0$ and $t^{\prime}=\epsilon z / \bar{w}$, following the general prescription given in Sec. III C, we find the nonholomorphic coordinate transformation,

$$
z^{\prime 1}=z^{1}\left(1+\left|z^{2}\right|^{2}\right), \quad z^{\prime 2}=-\epsilon \frac{z^{1} z^{2}}{\bar{z}^{1}},
$$

relating the metrics obtained from (98) and (100). This illustrates the general principle outlined in this paper.

\section{2. "Improved" version}

To obtain twistor lines that fall in the class discussed in Sec. II C, one can change coordinates to

$$
\xi=\frac{u_{2} u_{3}}{u_{1}}, \quad \widetilde{\xi}=\frac{1}{2} \log \frac{u_{3}}{u_{2}}, \quad \alpha=\frac{1}{2} \log u_{1} .
$$

This is a contact transformation, in the sense that

$$
\mathrm{d} u_{1}+u_{2} \mathrm{~d} u_{3}-u_{3} \mathrm{~d} u_{2}=2 u_{1}(\mathrm{~d} \alpha+\xi \mathrm{d} \tilde{\xi}) .
$$

In terms of these new Darboux coordinates, the twistor lines now read

$$
\xi=(z-\epsilon \bar{w} t)(w+\epsilon \bar{z} t) / t, \quad \widetilde{\xi}=\frac{1}{2} \log \frac{w+\epsilon \bar{z} t}{z-\epsilon \bar{w} t}, \quad \alpha=\frac{1}{2} \log t,
$$

which do fall in the class of (23). These expressions for the twistor lines are valid in the patch away from the cut from $t_{+}=\epsilon z / \bar{w}$ to $t_{-}=-\epsilon w / \bar{z}$.

This description follows from the following construction of the twistor space. We cover the twistor sphere by three patches: the patches $\mathcal{U}_{t_{ \pm}}$surround the points $t_{ \pm}$and the rest is covered by $\mathcal{U}_{0}$. The two nontrivial transition functions are given by

$$
H^{\left[0 t_{ \pm}\right]}= \pm \frac{1}{2} \xi \log \xi
$$

and there is a nonvanishing anomalous dimension $c_{\alpha}=\frac{1}{2}$. Then general equations (24) and (26) reproduce $^{42}$ twistor lines (104) if one identifies

$$
\mathcal{R}=|z w|, \quad A=\epsilon\left(|z|^{2}-|w|^{2}\right), \quad B=\arg \frac{w}{z}, \quad B_{\alpha}=\arg (z w),
$$

and rotates $t$ by the phase of $z w: t \rightarrow t \sqrt{\bar{z} \bar{w} / z w}$.

The Kähler potential, after a Kähler transformation, becomes

$$
K=\log [\cosh (\alpha+\bar{\alpha})+\epsilon|\xi| \cosh (\tilde{\xi}+\overline{\tilde{\xi}})],
$$

and the contact potential is now $t$ independent, 


$$
\mathrm{e}^{\Phi^{\prime}}=\mathrm{e}^{\Phi / u_{1}}=\frac{1}{4}\left(1+\epsilon\left(|z|^{2}+|w|^{2}\right)\right)
$$

Choosing the slice $t=0$, the procedure of Sec. III B 3 leads to

$$
z^{1}=\frac{1}{2} \log (z w), \quad z^{2}=\frac{1}{2} \log \frac{w}{z}
$$

and

$$
h=-2 \log \frac{1}{2}\left(\mathrm{e}^{-\left(z^{1}+\bar{z}^{1}\right)}+2 \epsilon \cosh \left(z^{2}+\bar{z}^{2}\right)\right) .
$$

\section{B. The universal hypermultiplet}

In this section, we illustrate our general results in the case of the perturbative hypermultiplet moduli space in type IIA string theory compactified on a rigid Calabi-Yau threefold $X$. For brevity and following a common abuse of language, we refer to this space as the "universal hypermultiplet" space $\mathcal{M}$. This is a (noncomplete) quaternion-Kähler deformation of the quaternion-Kähler symmetric space $\mathrm{SU}(2,1) / \mathrm{U}(2)$, a noncompact version of $\mathrm{C} P^{2}=\mathrm{SU}(3) / \mathrm{U}(2)$. The deformation is physically interpreted as a one-loop correction to the moduli space metric. It has a curvature singularity which is presumed to be resolved by instanton corrections (perturbative corrections vanish beyond one-loop).

The metric on $\mathcal{M}$ can be written in terms of four real coordinates, denoted by $\{r, \zeta, \widetilde{\zeta}, \sigma\}{ }^{43}$ as follows:

$$
\mathrm{d} s_{\mathrm{UHM}}^{2}=-\frac{3}{2 \Lambda r^{2}}\left[\frac{r+2 c}{r+c} \mathrm{~d} r^{2}+(r+2 c) \frac{|\mathrm{d} \tilde{\zeta}+(\tau / 2) \mathrm{d} \zeta|^{2}}{\operatorname{Im} \tau}+\frac{r+c}{16(r+2 c)}(\mathrm{d} \sigma+\widetilde{\zeta} \mathrm{d} \zeta-\zeta \mathrm{d} \widetilde{\zeta})^{2}\right] .
$$

Here $r=\mathrm{e}^{\phi}$ represents the four-dimensional string coupling constant $1 / g_{4}^{2}, \zeta / 2, \tilde{\zeta}$ are the periods of the Ramon-Ramond three-form on a basis of $H^{3}(\mathcal{X}, Z)$, and $\sigma$ is the Neveu-Schwarz axion. The parameter $\tau$ is the modulus of the Jacobian of $\mathcal{X}$ (see Ref. 12, for more details); it can be reabsorbed into a linear coordinate transformation of $(\zeta / 2, \widetilde{\zeta})$. Although it does affect the global structure of $\mathcal{M}$, for the local considerations in this work there is no loss of generality in setting $\tau=\mathrm{i}$. The parameter $c=-\chi_{X} / 192 \pi$ is determined by the one-loop correction to the tree-level metric, with $\chi_{X}$ the Euler number of the Calabi-Yau manifold $X .^{7}$ For $c=0$, or in the classical limit $r \rightarrow \infty$, (111) reduces to the metric on $\mathrm{SU}(2,1) / \mathrm{U}(2)$ with isometry group $\mathrm{SU}(2,1)$. For $c \neq 0$, the isometry group is broken to the three-dimensional Heisenberg group acting as shifts in $\sigma$, $\zeta$, and $\tilde{\zeta}$

$$
r \rightarrow r, \quad \zeta \rightarrow \zeta+\gamma, \quad \tilde{\zeta} \rightarrow \tilde{\zeta}+\beta, \quad \sigma \rightarrow \sigma-4 \alpha-\beta \gamma+\gamma \tilde{\zeta}-\beta \zeta
$$

where $\alpha, \beta$ and $\gamma$ are real parameters. Despite appearances, quaternion-Kähler metric (111) is regular at $r=0$ and $r=-c .{ }^{44}$ The curvature singularity at $r=-2 c$ is expected to be resolved by instanton corrections.

\section{Calderbank-Pedersen, Toda, and Przanowski}

Since the Killing vectors $\kappa_{1}=\partial_{\sigma}$ and $\kappa_{2}=\partial_{\tilde{\zeta}}-\zeta \partial_{\sigma}$ commute, the metric can be brought into the Calderbank-Pedersen form of Sec. IV B. This is achieved by changing coordinates to 7,10

$$
\tau=\sqrt{r+c}, \quad \eta=\frac{\zeta}{4}, \quad \varphi=\tilde{\zeta}, \quad \psi=\frac{1}{8}(\sigma+\zeta \widetilde{\zeta})
$$

The Calderbank-Petersen potential $\mathcal{P}$ and Toda potential $T$ are then given by 


$$
\mathcal{P}=\tau^{3 / 2}-c \tau^{-1 / 2}, \quad T=\log (\rho+c),
$$

where we have used the fact that $G=\tau^{2}-c, V=-2 \eta$ which are easily seen to satisfy (84) and (74).

From the general discussion in Sec. IV B, or referring back to Ref. 11, we can therefore cast metric (111) in Przanowski's form (1) by setting

$$
\begin{gathered}
z^{1}=-\left(r+c \log (r+c)-\frac{1}{8} \zeta^{2}\right)-\frac{\mathrm{i}}{4}(\sigma+\zeta \widetilde{\zeta}), \quad z^{2}=-\frac{1}{4}(\zeta-2 \mathrm{i} \tilde{\zeta}), \\
h=-\log \frac{r^{2}}{r+c} .
\end{gathered}
$$

In this formula, $r$ is the function of $\left(z^{1}, z^{2}\right)$ and is determined by the following equation:

$$
r+c \log (r+c)=\frac{1}{2}\left(z^{2}+\bar{z}^{2}\right)^{2}-\frac{1}{2}\left(z^{1}+\bar{z}^{1}\right) .
$$

By computing the derivatives of $h$ implicitly, it is easy to verify that (116) does solve master Eq. (1). For $c=0, h$ may be expressed explicitly in terms of $\left(z^{1}, z^{2}\right)$,

$$
c=0: \quad h=-\log \left[\frac{1}{2}\left(z^{2}+\bar{z}^{2}\right)^{2}-\frac{1}{2}\left(z^{1}+\bar{z}^{1}\right)\right]
$$

\section{Twistor space}

On the other hand, the twistor description for (111) is based on the covering of the Riemann sphere by three patches: ${ }^{19,20} \mathcal{U}_{ \pm}$are patches around the north and the south pole, whereas the patch $\mathcal{U}_{0}$ covers the rest. ${ }^{45}$ Specifying the general results of Refs. 19 and 20 to the prepotential $F(X)$ $=\frac{i}{4} X^{2}$, one obtains that the transition functions between these patches read

$$
H^{[0+]}=-\frac{1}{8} \xi^{2}, \quad H^{[0-]}=\frac{1}{8} \xi^{2} .
$$

In addition, $\alpha$ has anomalous dimension, due the one-loop correction,

$$
c_{\tilde{\xi}}=0, \quad c_{\alpha}=-2 c .
$$

They lead to the following Darboux coordinates in the patch $\mathcal{U}_{0},{ }^{46}$

$$
\begin{gathered}
\xi=\zeta+2 \sqrt{r+c}\left(t^{-1}-t\right), \quad \tilde{\xi}=\frac{\mathrm{i}}{2} \widetilde{\zeta}+\frac{1}{2} \sqrt{r+c}\left(t^{-1}+t\right), \\
\alpha+\frac{1}{2} \tilde{\xi} \tilde{\xi}=-\frac{\mathrm{i}}{4} \sigma-\frac{1}{4} \sqrt{r+c}\left((\zeta-2 \mathrm{i} \tilde{\zeta}) t^{-1}+(\zeta+2 \mathrm{i} \widetilde{\zeta}) t\right)-2 c \log t .
\end{gathered}
$$

Following the same procedure as in Sec. V A, one can compute the contact potential and the $\mathrm{SU}(2)$ connection one-forms. The contact potential is real, constant along the fiber, and coincides with the four-dimensional dilation,

$$
\mathrm{e}^{\Phi}=r
$$

so that the Kähler potential is given by

$$
K=\log \frac{2 r(1+t \bar{t})}{|t|} .
$$

It is impossible to write it explicitly as a function of Darboux coordinates and their complex conjugate. This can be done however for $c=0$, in which case it reads as ${ }^{47}$ 


$$
K=\frac{1}{2} \log \left[\left(\frac{1}{16}(\xi-\bar{\xi})^{2}-(\widetilde{\xi}+\overline{\tilde{\xi}})^{2}\right)^{2}-\left(\alpha+\bar{\alpha}+\frac{1}{2}(\xi+\bar{\xi})(\tilde{\xi}+\overline{\tilde{\xi}})\right)^{2}\right]
$$

One can explicitly check that Monge-Ampère equation (31) is satisfied, as well as the other identities on twistor space (30). The $\mathrm{SU}(2)$ connection is easily found by expanding the contact form,

$$
p^{+}=-\frac{\sqrt{r+c}}{4 r}(\mathrm{~d} \zeta-2 \mathrm{id} \widetilde{\zeta}), \quad p^{3}=\frac{1}{8 r}(\mathrm{~d} \sigma+\widetilde{\zeta} \mathrm{d} \zeta-\zeta \mathrm{d} \widetilde{\zeta}) .
$$

One may check that the Przanowski description presented above follows from this twistor construction by restricting to the $t=0$ slice. In particular, function (116) follows from (56) and coordinates (115) coincide with (55) up to a constant shift in $z^{1}$. It is also easy to verify that relations (81) and (89) to the Toda and the Calderbank-Pedersen variables, respectively, are indeed satisfied.

\section{Alternative Przanowski description}

Przanowski function (116) has a type 1 Killing vector $\kappa_{1}=\partial_{\sigma}$ and is canonically associated with $\kappa_{1}$ along the lines of Sec. IV A. The other Killing vector $\kappa_{2}=\partial_{\tilde{\zeta}} \zeta \partial_{\sigma}$ is realized as a type 2 isometry. There is, however, a different Przanowski representation of metric (111) canonically associated with $\kappa_{2}$, i.e., where $\kappa_{2}$ becomes a type 1 isometry. Since the moment map associated with $\kappa_{2}$ is proportional to $\xi$, this representation may be reached by choosing the complex submanifold $\xi=0$. More generally, the complex submanifold $\xi=x$ for any fixed complex number $x$ produces a Przanowski representation of metric (111), which interpolates between the two aforementioned representations. For simplicity, we restrict to the case with $c=0$.

The slice $\xi=x$ is an example of the $\hat{\xi}$-parametrization discussed in Sec. III B 1 and leads to the Przanowski description provided by (48) and (49) with $W=x \widetilde{\xi}$. This gives

$$
z^{\prime 1}=\widetilde{\xi}, \quad z^{\prime 2}=\alpha+x \tilde{\xi}
$$

and produces the following solution of the master equation:

$$
h^{\prime}\left(z^{\prime 1}, z^{\prime 2}\right)=-\log \left[\left(\left(z^{\prime 1}+\bar{z}^{\prime 1}\right)^{2}-\frac{(x-\bar{x})^{2}}{16}\right)^{2}-\left(z^{\prime 2}+\bar{z}^{\prime 2}-\frac{x-\bar{x}}{2}\left(z^{\prime 1}-\bar{z}^{\prime 1}\right)\right)^{2}\right] .
$$

The coordinates $z^{\prime \alpha}$ are related to the real coordinates $x^{\mu}$ in (111) by solving the condition $\xi=0$ for $t\left(x^{\mu}\right)$ and plugging into (126),

$$
\begin{gathered}
z^{\prime 1}=\frac{\mathrm{i}}{2} \widetilde{\zeta}+\frac{1}{4} \sqrt{16 r+(\zeta-x)^{2}}, \\
z^{\prime 2}+x z^{\prime 1}=-\frac{\mathrm{i}}{4}(\sigma+\zeta \widetilde{\zeta})-\frac{1}{8}(\zeta+x) \sqrt{16 r+(\zeta-x)^{2}} .
\end{gathered}
$$

In particular, as advertised previously, the Killing vector $\kappa_{2}$ is now type 1 . The fact that $h^{\prime}$ is independent of $x+\bar{x}$ follows from the fact that $\operatorname{Re}(x)$ can be reabsorbed into a shift of $\zeta$. In particular, for $x=0$, coordinates (129) are related to (115) by the nonholomorphic change of variables,

$$
z^{\prime 1}=\frac{1}{2}\left(\bar{z}^{2}-z^{2}\right)+\frac{1}{4} \sqrt{16\left(z^{1}+\bar{z}^{1}\right)+12\left(z^{2}+\bar{z}^{2}\right)^{2}},
$$




$$
z^{\prime 2}=\left(\bar{z}^{1}-z^{1}\right)-\frac{2\left(z^{2}+\bar{z}^{2}\right)}{8} \sqrt{16\left(z^{1}+\bar{z}^{1}\right)+12\left(z^{2}+\bar{z}^{2}\right)^{2}},
$$

which illustrates the general situation described in Sec. III C.

\section{LINEAR PERTURBATIONS}

In this section, we present one of the main results of the paper. Namely, we analyze linear perturbations of self-dual Einstein manifolds with an isometry. The general theory of quaternionic perturbations was developed in Ref. 19, which we specify here to the case of four dimensions. We show that linearized master equation (3) is equivalent, after suitable rescaling of $\delta h$, to the vanishing of the conformal Laplace-Beltrami operator on $\mathcal{M}$. We further express the variations $\delta h$ through the Penrose-type integral of a set of holomorphic functions representing a section of $H^{1}(\mathcal{Z}, \mathcal{O}(2))$, which governs the perturbations of the twistor space $\mathcal{Z}$.

\section{A. Linearized master equation and conformal massless field}

To begin, we observe that in the background of self-dual Einstein manifold $\mathcal{M}$ with a type 1 isometry, linearized master equation (3) controlling perturbations of the quaternion-Kähler metric on $\mathcal{M}$ has a simple relation to the Laplace-Beltrami operator on $\mathcal{M}$. Indeed, noting that the inverse Przanowski metric is given, in $\partial_{1}, \partial_{2}$ basis, by

$$
g^{\alpha \bar{\beta}}=-\frac{\Lambda}{3} \frac{\mathrm{e}^{-h}}{\left|h_{1}\right|^{2}}\left(\begin{array}{cc}
h_{2 \overline{2}}+2 \mathrm{e}^{h} & -h_{2 \overline{1}} \\
-h_{1 \overline{2}} & h_{1 \overline{1}}
\end{array}\right),
$$

the Laplace-Beltrami operator on $\mathcal{M}$ reads

$$
\Delta=\frac{1}{\sqrt{g}} \partial_{\mu}\left(\sqrt{g} g^{\mu \nu} \partial_{\nu}\right)=-\frac{2 \Lambda \mathrm{e}^{-h}}{3\left|h_{1}\right|^{2}}\left[h_{1 \overline{1}} \partial_{2 \overline{2}}+h_{2 \overline{2}} \partial_{1 \overline{1}}-h_{12} \partial_{2 \overline{1}}-h_{21} \partial_{1 \overline{2}}+\mathrm{e}^{h}\left(h_{1} \partial_{1}^{-}+h_{1}^{-} \partial_{1}+2 \partial_{11}\right)\right]
$$

It is then a simple matter to check that, when $h_{1}=h_{1}^{-}$,

$$
\operatorname{dPrz}_{h}(\delta h)=\mathrm{e}^{h}\left|h_{1}\right|^{4}\left[-\frac{3}{2 \Lambda} \Delta+1\right] \frac{\delta h}{\left|h_{1}\right|^{2}} .
$$

Thus, in this case, the metric perturbations preserving the QK property (but not the isometries) are, up to rescalings by $\left|h_{1}\right|^{2}$, eigenmodes of the Laplace-Beltrami operator, with a specific eigenvalue equal to $R / 6=2 \Lambda / 3$. This value corresponds to a conformally coupled massless scalar field, such that (133) is invariant under conformal rescalings of the background metric. ${ }^{20}$

In case $h_{1} \neq h_{1}^{-}$, a more general statement holds for any solution of the master equation, namely,

$$
\operatorname{dPrz}_{h}(\delta h)=\mathrm{e}^{h}\left|h_{1}\right|^{4}\left[-\frac{3}{2 \Lambda} \tilde{\Delta}+1\right] \frac{\delta h}{\left|h_{1}\right|^{2}},
$$

where the operator

$$
\tilde{\Delta}=\Delta+2 \mathcal{V}_{\mu} g^{\mu \nu}\left(\partial_{\nu}+\frac{1}{2}\left(\partial_{\nu} \log \left|h_{1}\right|^{2}\right)\right), \quad \mathcal{V}_{\alpha}=\partial_{\alpha} \log \frac{h_{1}}{h_{1}^{-}},
$$

reduces to $\Delta$ when $h_{1}=h_{1}^{-}$. However, the geometric interpretation of this more general operator is unclear in the absence of type-1 isometries. 


\section{B. Penrose contour integrals}

As explained e.g., in Refs. 20, 48, and 49, eigenmodes of the Laplace-Beltrami operator ${ }^{50}$ on a quaternion-Kähler manifold with this particular eigenvalue are known to be generated by a Penrose-type contour integral formula ${ }^{20}$ from a holomorphic section of $H^{1}(\mathcal{S})$, homogeneous of degree 2 . This section may be described locally by holomorphic functions $\hat{\Psi}^{[i j]}$, subject to the cocycle relation $\hat{\Psi}^{[i j]}+\hat{\Psi}^{[j k]}=\hat{\Psi}^{[i k]}$. The solution $\psi$ is then obtained by the contour integral formula,

$$
\psi=\frac{1}{2 \pi \mathrm{i}} \sum_{j} \oint_{C_{j}} \pi_{A^{\prime}} D \pi^{A^{\prime}} \hat{\Psi}^{[i j]}\left(\pi, x^{\mu}\right), \quad\left[-\frac{3}{2 \Lambda} \Delta+1\right] \psi=0,
$$

where $C_{j}$ are closed contours around each of the patches $\mathcal{U}_{j}$ and $\pi_{A^{\prime}} D \pi^{A^{\prime}}$ is the Liouville form on $\mathcal{S}$ (note that the right hand side is independent of the value of $i$ due to the cocycle relations.). Using (19), it is easily seen to be equal to

$$
\pi_{A^{\prime}} D \pi^{A^{\prime}}=\mathrm{e}^{2 \lambda^{[j]}} \mathcal{X}^{[j]}=2 \mathrm{e}^{2 \lambda^{[j]}+\Phi^{[j]}} \frac{D t}{t} .
$$

Moreover, since $\hat{\Psi}^{[i j]}\left(\pi, x^{\mu}\right)$ is holomorphic and homogeneous of degree 2, it can be expressed as $\hat{\Psi}^{[i j]}\left(\pi, x^{\mu}\right)=\mathrm{e}^{-2 \lambda^{[j]}} \Psi^{[i j]}(\xi, \widetilde{\xi}, \alpha)$, where $\Psi^{[i j]}(\xi, \widetilde{\xi}, \alpha)$ can be viewed as an element of $H^{1}(\mathcal{Z}, \mathcal{O}($ -2))). Putting these facts together, the Penrose integral (136) may be rewritten as

$$
\psi=2 \sum_{j} \oint_{C_{j}} \frac{\mathrm{d} t}{2 \pi \mathrm{i} t} \mathrm{e}^{\Phi^{[j]}} \Psi^{[i j]}(\xi, \widetilde{\xi}, \alpha),
$$

where $\Phi, \xi, \widetilde{\xi}, \alpha$ are viewed as meromorphic functions of $t$ for a fixed point $x^{\mu}$ on the base $\mathcal{M}$. In the case of an Einstein self-dual metric with a type 1 isometry, this may be rewritten using (69) as

$$
\psi=h_{1}^{-1} \sum_{j} \oint_{C_{j}} \frac{\mathrm{d} t}{2 \pi \mathrm{i} t} \Psi^{[i j]}(\xi, \tilde{\xi}, \alpha)
$$

On the other hand, we know from Refs. 19 and 51 that deformations of the twistor space $\mathcal{Z}$ are governed by an element of $H^{1}(\mathcal{Z}, \mathcal{O}(2))$. This can be represented locally by holomorphic functions $H_{(1)}^{[i j]}(\xi, \widetilde{\xi}, \alpha)$, defined on the overlap of two patches $\mathcal{U}_{i} \cap \mathcal{U}_{j}$, which determine the perturbation of the holomorphic transition function $H^{[i j]}$ from Sec. II B. The perturbed contact potential is then obtained by expanding (25) and (26) to linear order in perturbation. In the case when the background has one isometry, we can use results (56), (55), and (71) to obtain the perturbation of the Przanowski function,

$$
\delta h=-2 \delta \phi-h_{\alpha} \delta z^{\alpha}-h_{\bar{\alpha}} \delta \bar{z}^{\bar{\alpha}}=-\mathrm{e}^{-\phi}\left[2 \delta \mathrm{e}^{\phi}+\left(\operatorname{Re} \delta \alpha_{0}^{[+]}+\xi_{0}^{[+]} \delta \widetilde{\xi}_{0}^{++]}\right)\right] .
$$

Keeping the real coordinates $\mathcal{R}, A, B, B_{\alpha}$ unperturbed and using integral equations (24), one finds

$$
\delta h=-h_{1} \sum_{j} \int_{C_{j}} \frac{\mathrm{d} t}{2 \pi \mathrm{i} t}\left(H_{(1)}^{[+j]}+\delta \tilde{\xi}^{\tilde{j}]]} \partial_{\xi j]}\left[H^{[+j]}-\left(\xi^{[+]}-\xi_{(0)}\right) \delta \widetilde{T}^{+j]}\right),\right.
$$

where $\xi_{(0)}=A+\mathcal{R}\left(t^{-1}-t\right)$. This can be further simplified by substituting integral representations for $\delta \tilde{\xi}$ and $\xi-\xi_{(0)}$. Then the second and third terms differ essentially only by the order of integrations. They exactly cancel each other since the contribution of the pole at $t=t^{\prime}$ coming from changing the order of integration is canceled by the term accounting for the difference between $\xi^{[+]}$in (141) and $\xi^{[j]}$ to be used in this cancellation. Thus, one finally obtains a very simple result, ${ }^{52}$ 


$$
\delta h=-h_{1} \sum_{j} \oint_{C_{j}} \frac{\mathrm{d} t}{2 \pi \mathrm{i} t} H_{(1)}^{[i j]} .
$$

Thus, comparing (142) with (139) and using (133), we may identify

$$
\psi=\frac{\delta h}{\left|h_{1}\right|^{2}}, \quad \Psi^{[i j]}=-H_{(1)}^{[i j]} .
$$

This identification between $H^{1}(\mathcal{Z}, \mathcal{O}(2))$ and $H^{1}(\mathcal{Z}, \mathcal{O}(-2))$ of course relies on the particular trivialization $\lambda=0$ chosen to relate $\hat{\Psi}$ and $\Psi$. Using (143), we can in principle lift any solution of the linearized master equation (3) to a section of $H^{1}(\mathcal{Z}, \mathcal{O}(2))$, and therefore infer the contact structure on the deformed twistor space.

\section{Instanton corrections to the universal hypermultiplet}

To illustrate the above results, we now make contact with the analysis of instanton corrections to the universal hypermultiplet in Ref. 11. To begin, however, note that for the metric (111), one may directly prove that contour integral (139) produces eigenmodes of the conformal Laplacian, using the facts that $h_{1}=1 /(2 r)$ and

$$
\left.\left[-\frac{3}{2 \Lambda} \Delta+1-\partial_{t}\left(\frac{r^{2}}{4(r+c)(r+2 c)}\left(t^{2} \partial_{t}+t\right)+4 \mathrm{i} t \frac{r^{2}}{r+c} \partial_{\sigma}\right)\right)\right]\left(\frac{r}{t} \Psi\right)=0
$$

for any holomorphic function $\Psi(\xi, \widetilde{\xi}, \alpha)$. If $C_{j}$ are closed contours, one may integrate by parts and check that conformal Laplace equation (136) is satisfied. One may also consider open contours, provided one makes sure that all boundary contributions coming from the integration by parts and the action of the Laplace operator on the limits of integration cancel each other. It can be demonstrated by a rather lengthy computation that this is the case when the end points of the contour lie on any complex submanifold of $\mathcal{Z}{ }^{53}$ Such open contours will play an important role in what follows.

As a first example, we choose a set of holomorphic functions independent of $\alpha$ of the form

$$
\Psi_{p, q}(\xi, \widetilde{\xi})=\mathrm{e}^{\mathrm{i} q \xi-2 p \tilde{\xi}},
$$

for arbitrary "charges" $p, q$. We integrate it over the contour $C$ going from $t=0$ to $t=\infty$ along the direction $(p-(\mathrm{i} / 2) q) t^{-1} \in \mathbb{R}^{+}$

$$
\delta h_{p, q}=h_{1} \int_{0}^{\infty} \frac{\mathrm{d} t}{2 \pi \mathrm{i} t} \mathrm{e}^{\mathrm{i} q \tilde{\xi}-2 p \tilde{\xi}}
$$

By using (144), this will produce an eigenmode of the conformal Laplacian; the boundary terms vanish since the exponential is exponentially suppressed at $t=0, \infty$ along $C$. The integral (146) can now easily be done and produces a modified Bessel function with index 0 ,

$$
\delta h_{p, q}=\frac{\mathrm{e}^{i(q \zeta-p \tilde{\zeta})}}{4 \pi \mathrm{i} r} K_{0}\left(2 \sqrt{(r+c)\left(4 q^{2}+p^{2}\right)}\right) .
$$

In Ref. 11, this deformation was found to describe a single D-brane instanton ${ }^{54}$ correction, coming from a wrapped D2-brane on the cycle $(p, q) \in H_{3}(X, Z)$. The same holomorphic function as in (145) also governs the complex contact transformations on $\mathcal{Z}$ and, in particular, determines the D2-brane corrections to contact potential (26) in terms of a modified Bessel function with index $1 .^{23}$ It is straightforward to check that this is indeed consistent with formula (56) relating the contact potential and the Przanowski function in the special slice $t=0$. In checking this one must take into account the deformation of the coordinates $z^{\alpha}$ as in (140).

As a second example, we consider, for $k>0$ and $\nu \in \mathbb{C}$, 


$$
\Psi_{k, \pm}^{(\nu)}=\left(\frac{1}{4} \xi \pm \widetilde{\xi}\right)^{\nu} \mathrm{e}^{\mp 4 k(\alpha+(1 / 2) \tilde{\xi})-k\left((1 / 4) \xi^{2}-4 \tilde{\xi}^{2}\right)},
$$

and choose a contour $C$ connecting $t=\infty$ (for $\Psi_{k,+}$, respectively, $t=0$ for $\left.\Psi_{k,-}\right)$ to the point $t_{ \pm}\left(x^{\mu}\right)$ on the complex submanifold $\xi \pm 4 \widetilde{\xi}=0$, namely,

$$
t_{ \pm}=-\left[\frac{4 \sqrt{r+c}}{ \pm \zeta+2 \mathrm{i} \tilde{\zeta}}\right]^{ \pm 1}
$$

Changing the integration variable as $t=t_{ \pm}(s+1)^{ \pm 1}$, one finds

$$
\delta h_{k, \pm}^{(\nu)}=C \frac{\left(\frac{1}{4} \zeta \pm \frac{\mathrm{i}}{2} \tilde{\zeta}\right)^{\nu-8 c k}}{r \sqrt{r+c}} W_{4 c k-\nu-(1 / 2), 4 c k}(8 k(r+c)) \mathrm{e}^{-k\left((1 / 4) \zeta^{2}+\tilde{\zeta}^{2}\right) \pm i k \sigma}
$$

where $C$ is an irrelevant constant and the Whittaker function is defined as the integral,

$$
W_{\ell, m}(z)=\frac{\mathrm{e}^{-z / 2} z^{m+1 / 2}}{\Gamma\left(\frac{1}{2}-\ell+m\right)} \int_{0}^{\infty} s^{m-\ell-1 / 2}(s+z)^{m+\ell-1 / 2} \mathrm{e}^{-z s} \mathrm{~d} s .
$$

This result reproduces the family of solutions discussed in Eq. B19 in Ref. 11 for $\kappa=8 c k-\nu$. In the weak coupling limit $r \rightarrow \infty, \delta h_{k, \pm}^{(\nu)}$ is exponentially suppressed,

$$
\delta h_{k, \pm}^{(\nu)} \sim \frac{\left(\frac{1}{4} \zeta \pm \frac{\mathrm{i}}{2} \tilde{\zeta}\right)^{\nu-8 c k}}{r(r+c)^{1+\nu-8 c k}} \mathrm{e}^{-4 k r-k\left((1 / 4) \zeta^{2}+\tilde{\zeta}^{2}\right) \pm i k \sigma}
$$

and the argument of the exponential agrees with the NS5-brane classical action. Note that (150) has branch cuts in the $(\zeta, \widetilde{\zeta})$ plane, a feature which may be undesirable for NS5-brane instanton corrections. However, for the special value $\nu=8 c k$ in (150), the branch cut disappears and the instanton correction becomes particularly simple. In this case it can be written also through the incomplete Gamma function,

$$
\delta h_{k, \pm}^{(8 c k)}=C r^{-1}(r+c)^{4 c k} \Gamma(-8 c k, 8 k(r+c)) \mathrm{e}^{4 k r-k\left((1 / 4) \zeta^{2}+\tilde{\zeta}^{2}\right) \pm i k \sigma},
$$

where $\Gamma(s, x)=\int_{x}^{\infty} t^{s-1} \mathrm{e}^{-t} \mathrm{~d} t$ is the incomplete gamma function. This reproduces the solution (4.6) in Ref. 11. For $c=0$ it also agrees with Eq. (4.72) of Ref. 12 upon setting $s=\ell_{1}=\ell_{2}$ in this reference.

More generally, one would like to determine the holomorphic functions corresponding to a complete basis of solutions for the perturbations around the universal hypermultiplet satisfying (3), regular in the $\zeta$ plane, and exponentially decaying at $r=\infty$ and $\zeta=\infty$. A basis of solutions satisfying these boundary conditions can be found by separation of variables and is given, in the sector with nonzero momentum along the $\sigma$-direction, by

$$
\delta h_{k, n, \pm}=r^{-1}(r+c)^{4 c k} H_{n}(\zeta \sqrt{k}) U(1+n+8 c k, 1+8 c k, 8 k(r+c)) \mathrm{e}^{-4 k r-k\left(\zeta^{2} / 2\right) \pm i k(\sigma+\zeta \tilde{\zeta})},
$$

where $k>0, n \in \mathbb{N}, H_{n}$ are the Hermite polynomials and $U$ is the confluent hypergeometric function of the second kind. For $n=0$, this reduces to Eq. (4.9) in Ref. 11, while for $c=0$, it reduces to the non-Abelian Fourier eigenmodes in Eq. (1.18) of Ref. 12, again setting $s=0, n=0$ in this reference. We have found a representation of the holomorphic section $\Psi_{k, n}$ governing (154) in the special case $n=0$ and hope to address the general case elsewhere.

\section{DISCUSSION}

In this work, we have discussed Einstein self-dual manifolds $\mathcal{M}$, the four-dimensional avatar of quaternion-Kähler manifolds, with particular emphasis on their heavenly description, namely as 
solutions of Przanowski's equation (1). In particular, we have related this description to the more standard twistor construction for quaternion-Kähler manifolds and have shown that the Przanowski function $h$ was equal to the Kählerpotential $K$ on $\mathcal{Z}$ in a certain Kähler gauge, restricted to any complex (local) submanifold $\mathcal{C}$ of $\mathcal{Z}$, Eq. (45) above. Different choices of $\mathcal{C}$ lead to diffeomorphic Hermitian metrics on $\mathcal{M}$, where different (local, integrable) complex structures are manifest. Varying $\mathcal{C}$ leads to "pure gauge" solutions of linearized master equation (3), which lie outside the class of infinitesimal holomorphic diffeomorphisms (5). These "pure gauge" solutions, however, do not seem to be expressible in terms of $h$ and its derivatives in general, see (66) and (A14) for two illustrative examples.

If $\mathcal{M}$ admits a Killing vector, there is a preferred choice of submanifold $\mathcal{C}$, the zero locus of the $\mathcal{O}(2)$ valued moment map, which determines a canonical complex structure (up to complex conjugation). In this complex structure, the Przanowski function has a type 1 symmetry $\left(h_{1}=h_{1}^{-}\right)$ and determines a solution of continuous Toda equation (74) via (79). This reproduces Tod's parametrization (72) of Einstein self-dual manifolds with one isometry. If $\mathcal{M}$ admits two commuting isometries, then we have shown that it could be represented by a Przanowski function with both type 1 and type $2\left(h_{2}=h_{2}\right)$ symmetries. Such a function determines a solution of the Laplace equation on the Poincaré upper half-plane (84), reproducing the Calderbank-Petersen parametrization (83) of Einstein self-dual manifolds with two commuting isometries. To our knowledge, the relation between the Calderbank-Petersen potential $\mathcal{P}$ and the Toda potential $T$ has not appeared previously in the literature.

By a similar reasoning, a Killing tensor of higher rank on $\mathcal{M}$ would determine a $2 n$-plet of locally integrable complex structures. Indeed, for a rank $n$ Killing tensor, there is a variant of the moment map construction, which is now a $\mathcal{O}(2 n)$ global section $\mu .^{55,56}$ Any of the $2 n$ zeros of $\mu$ determines a locally integrable complex structure. It would be interesting to understand how the generalized Killing symmetry constrains the corresponding Przanowski function.

Having understood the twistorial origin of the Heavenly description, we were able to cast solutions of linearized master equation (3) around an Einstein self-dual metric with one Killing vector into the general formalism for perturbations of quaternion-Kähler manifolds developed in Ref. 11. To this aim, we observed that the linearized master equation around such a background is equivalent to the conformal Laplace-Beltrami operator on $\mathcal{M}$, Eq. (133), the zero modes of which can be obtained by a Penrose-type contour integral (138) of an holomorphic section $\Psi$ $\in H^{1}(\mathcal{Z}, \mathcal{O}(-2))$. By studying perturbations of the twistor lines on the special complex locus $t$ $=0$, we were able to relate $\Psi$ to the holomorphic section $H_{(1)} \in H^{1}(\mathcal{Z}, \mathcal{O}(2))$ which governs the perturbations of the complex contact structure on $\mathcal{Z}$. This relation in principle enables us to lift any solution of (3) to a perturbation of the twistor space $\mathcal{Z}$.

For perturbations around self-dual Einstein manifolds without isometry, the equivalence between the linearized master equation and the conformal Laplace-Beltrami operator no longer holds. Instead, one must replace the Laplace-Beltrami operator by $\widetilde{\Delta}$, defined in (135). It would be interesting to understand $\widetilde{\Delta}$ geometrically and provide a Penrose-type integral formula for general solutions of quaternionic perturbations where (134) vanishes.

The main motivation for this work was to understand the structure of instanton corrections in hypermultiplet moduli spaces in string theory. In Sec. VI we applied our results to the special case of the "universal hypermultiplet," i.e., the hypermultiplet moduli space in type IIA string theory compactified on a rigid Calabi-Yau manifold $X$, a particular example of a self-dual Einstein space with negative curvature. In particular, we showed that solutions (147) of the linearized master equation corresponding to D2-brane instantons are consistent with the analysis in Ref. 23. We have also taken some steps in lifting the solution (154), which should physically correspond to NS5-brane solutions, to the twistor space. We have succeeded for the related "symmetric gauge" solution (153). Such a solution (for $c=0$ ) appears in the Fourier expansion of the Picard Eisenstein series which was considered I Ref. 12, although it is not well suited for a systematic analysis of the non-Abelian Fourier expansion.

Eventually, one would like to be able to construct the exact quantum corrected metric on the hypermultiplet moduli space in rigid Calabi-Yau compactifications. This could be achieved by 
determining the exact contact structure on the twistor space, by providing a set of complex contact transformations consistent with the cocycle condition, such that the metric reduces to (111) in the weak coupling limit $r \rightarrow \infty$, up to exponentially suppressed corrections of the form discussed above. This finite deformation of (111) should tame the divergence of the D-instanton series ${ }^{57}$ and resolve the curvature singularity at $r=-2 c$. When $\tau=\sqrt{-d}$ where $d$ a positive integer, it was argued in Ref. 12 that this exact metric would be determined by automorphy under the Picard modular group $\mathrm{SU}(2,1, \mathbb{Z}[\tau])$. The analysis of Ref. 12 , however, remained suggestive only due to the difficulties of implementing discrete symmetries at the level of twistor space [see Ref. 44, for recent progress in the case of $S L(2, Z)]$. Hopefully the results in this paper will be useful in this direction.

More ambitiously, one would like to determine the exact quantum corrected metric on the hypermultiplet moduli space for general, nonrigid Calabi-Yau compactifications. In this respect, we note that the heavenly parametrization generalizes straightforwardly to quaternion-Kähler manifolds with dimension $4 n>4$, although master equation (1) is replaced by $2 n-1$ partial differential equations. It would be interesting to determine the linearized perturbations corresponding to NS5-brane instantons and their lift to the twistor space.

\section{ACKNOWLEDGMENTS}

We are grateful to D. Persson for valuable discussions, and to A. Neitzke and F. Saueressig for past collaboration on closely related topics. S.V. is grateful to LPTHE and LPTA for hospitality while this project was completed.

\section{APPENDIX A: DERIVATION OF THE PRZANOWSKI EQUATION IN THE $\xi=0$ GAUGE}

The complex slice $\xi=0$ allows for a simple "top-down" derivation of the Przanowski equation from the twistor space constraints as follows. Equations (33) reduce to

$$
K^{\tilde{\xi} \bar{\alpha}}=2 \mathrm{e}^{2 K} K_{\tilde{\xi}}, \quad K^{\xi \bar{\alpha}}=-2 \mathrm{e}^{2 K} K_{\tilde{\xi}}, \quad K^{\alpha \bar{\alpha}}=\mathrm{e}^{2 K} .
$$

Using the comatrix formula for $K_{\overline{\xi \xi}}^{\bar{\xi}}$ in terms of $K^{i \bar{j}}$ and Monge-Ampére equation (31), we readily obtain

$$
K^{\bar{\xi} \bar{\xi}}=4 \mathrm{e}^{2 K}\left(K_{\tilde{\xi} \bar{\xi}}+K_{\tilde{\xi}} K_{\bar{\xi}}\right) .
$$

Requiring that $K^{i \bar{j}} K_{\bar{j} k}=\delta_{k}^{i}$ produces four linear equations in $K_{\bar{\xi} \overline{\tilde{\xi}}}, K^{\bar{\xi} \overline{\tilde{\xi}}}, K_{\bar{\alpha} \xi}, K_{\bar{\xi}}$,

$$
\begin{aligned}
& \mathrm{e}^{2 K} K_{\alpha} \overline{\tilde{\xi}}-2 \mathrm{e}^{2 K} K_{\bar{\xi}} K_{\bar{\xi}} \overline{\tilde{\xi}}+2 \mathrm{e}^{2 K} K_{\bar{\xi}} K_{\bar{\xi} \bar{\xi}}=0, \\
& \mathrm{e}^{2 K} K_{\alpha \bar{\alpha}}-2 \mathrm{e}^{2 K} K_{\overline{\tilde{\xi}}} K_{\bar{\xi} \bar{\alpha}}+2 \mathrm{e}^{2 K} K_{\bar{\xi}} K_{\tilde{\xi} \bar{\alpha}}-1=0, \\
& -2 \mathrm{e}^{2 K} K_{\tilde{\xi}} K_{\alpha \bar{\alpha}}+4 \mathrm{e}^{2 K} K_{\bar{\xi} \bar{\alpha}}\left(K_{\tilde{\xi} \bar{\xi}}+K_{\tilde{\xi}} K_{\tilde{\xi}}\right)+K_{\tilde{\xi} \bar{\alpha}} K^{\overline{\tilde{\xi}}}=0, \\
& -2 \mathrm{e}^{2 K} K_{\tilde{\xi}} K_{\alpha} \overline{\bar{\xi}}+4 \mathrm{e}^{2 K} K_{\tilde{\xi} \overline{\tilde{\xi}}}\left(K_{\tilde{\xi} \bar{\xi}} \overline{\bar{\xi}}+K_{\tilde{\xi}} K_{\overline{\tilde{\xi}}}\right)+K_{\tilde{\xi} \bar{\xi}} K^{\bar{\xi} \overline{\tilde{\xi}}}=0 .
\end{aligned}
$$

A nonzero solution exists when the discriminant vanishes,

$$
\mathrm{e}^{2 K} K_{\alpha \overline{\tilde{\xi}}} K_{\tilde{\xi} \bar{\alpha}}+K_{\tilde{\xi} \bar{\xi}} \overline{\tilde{\xi}}+K_{\tilde{\xi}} K_{\overline{\tilde{\xi}}}-\mathrm{e}^{2 K} K_{\alpha \bar{\alpha}} K_{\bar{\xi} \bar{\xi}}^{\bar{\xi}}=0 .
$$

This reproduces the Przanowski equation upon identifying 


$$
z^{1}=\tilde{\xi}, \quad z^{2}=\alpha, \quad h=-2 K .
$$

Having imposed (A7) and setting $z^{3}=\xi$, we can now solve for the remaining components in terms of derivatives of $h$ in $z^{1}, z^{2}$ and of $K_{3}$ and $K_{3}^{-}$,

$$
\begin{gathered}
K_{3 \overline{1}}=\frac{h_{21}^{-}}{2 h_{1}^{-}}-\frac{h_{11}}{h_{1}^{-}} K_{3}^{-}, \\
K_{3 \overline{2}}=\frac{h_{2 \overline{2}}+2 \mathrm{e}^{h}}{2 h_{1}^{-}}+\frac{h_{1 \overline{2}}}{h_{1}^{-}} K_{3}^{-}, \\
K_{3 \overline{3}}=-\frac{1}{\left|h_{1}\right|^{2}}\left(\frac{1}{2} h_{22}+\mathrm{e}^{h}+K_{3} h_{2 \overline{1}}+K_{3}^{-} h_{1 \overline{2}}+2 h_{1 \overline{1}}\left|K_{3}\right|^{2}\right), \\
K^{1 \overline{1}}=-\frac{\mathrm{e}^{-h}}{\left|h_{1}\right|^{2}}\left(\frac{1}{2} h_{2 \overline{1}}^{-}+\mathrm{e}^{h}+K_{3} h_{2 \overline{1}}+K_{3}^{-} h_{1 \overline{2}}+\left(2 h_{1 \overline{1}}-\left|h_{1}\right|^{2}\right)\left|K_{3}\right|^{2}\right) .
\end{gathered}
$$

The functions $K_{3}$ and $K_{3}^{-}$are undetermined at this stage, however, additional conditions follow from requiring that $K_{3 \overline{3}}, K_{31}, K_{32}$ are derivatives of $K$. In particular, the phase $K_{3} / K_{3}^{-}$can be determined as follows. Since $h$ is related to the Kähler potential as in (49) with $W=0$, the variation of $W$ around zero should produce an eigenmode of linearized master equation (3). Therefore,

$$
\mathrm{dPrz}_{h}\left[-2 \delta W_{1}\left(z^{1}\right) K_{3}-\delta W\left(z^{1}\right) h_{2}\right]=0
$$

for any holomorphic function $W\left(z^{1}\right)$. Requiring the vanishing of the term proportional to $W^{\prime \prime}\left(z^{1}\right)$ leads to

$$
\mathrm{e}^{h} h_{1}^{-} K_{3}-\left(2 \mathrm{e}^{h}+h_{22}\right) K_{31}+h_{21} K_{32}=0,
$$

which reduces, using (A9), to

$$
\frac{K_{3}}{K_{3}^{-}}=\frac{h_{1}}{\bar{h}_{1}} .
$$

The vanishing of the term proportional to $W^{\prime}$ provides an additional constraint, but does not seem to allow to solve for the modulus of $K_{3}$ algebraically.

\section{APPENDIX B: RECONSTRUCTING THE TWISTOR LINES FROM PRZANOWSKI’S FUNCTION}

In the presence of two commuting isometries, and assuming for simplicity that the anomalous dimensions vanish, the twistor lines on $\mathcal{Z}$ can be found from the Przanowski function $h$ as follows. The Darboux coordinate $\xi$ is defined globally by the $\mathcal{O}(2)$-valued moment map for the type 2 isometry, Eq. (70). Using (71), this can be written as

$$
\xi=\frac{h_{2}}{h_{1}}+\frac{\mathrm{e}^{h / 2}}{h_{1}}\left(t^{-1}-t\right) .
$$

The other Darboux coordinates can be searched for as Taylor series in $t$, 


$$
\tilde{\xi}^{[+]}=z^{2}+\sum_{n=1}^{\infty} t^{n} \widetilde{\xi}_{n}^{[+]}, \quad \alpha^{[+]}=z^{1}+\sum_{n=1}^{\infty} t^{n} \alpha_{n}^{[+]} .
$$

Plugging in these expansions into (14) and using (71), (69), and (55), one may derive the following condition:

$$
\begin{aligned}
\mathrm{d}\left(z^{1}\right. & \left.+\sum_{n=1}^{\infty} t^{n} \alpha_{n}^{[+]}\right)+\frac{h_{2}+\mathrm{e}^{h / 2}\left(t^{-1}-t\right)}{h_{1}} \mathrm{~d}\left(z^{2}+\sum_{n=1}^{\infty} t^{n} \widetilde{\xi}_{n}^{[+]}\right)=\frac{1}{h_{1} t}\left(\mathrm{~d} t+\mathrm{e}^{h / 2} \mathrm{~d} z^{2}+\frac{t}{2}\right) h_{\alpha} \mathrm{d} z^{\alpha}-h_{\bar{\alpha}} \mathrm{d} \bar{z}^{\bar{\alpha}}( \\
& \left.+t^{2} e^{h / 2} \mathrm{~d} \bar{z}^{\bar{\alpha}}\right)
\end{aligned}
$$

Extracting the terms proportional to $\mathrm{d} t$, one obtains a set of algebraic equations which can be solved for $\alpha_{n}^{[+]}$, giving

$$
\alpha_{n}^{[+]}=-\frac{h_{2}}{h_{1}} \widetilde{\xi}_{n}^{[+]}+\frac{\mathrm{e}^{h / 2}}{n h_{1}}\left((n-1) \widetilde{\xi}_{n-1}^{++]}-(n+1) \widetilde{\xi}_{n+1}^{[+]}\right)
$$

The remaining terms then produce a sequence of differential equations for the coefficients $\tilde{\xi}_{n}^{[+]}$,

$$
\begin{gathered}
\mathrm{d}\left(\widetilde{\xi}_{2}^{+]}+z^{2}+\bar{z}^{2}\right)+\widetilde{\xi}_{2}^{[+]}\left(\mathrm{d} h-2 \mathrm{~d} \log h_{1}\right)+\mathrm{e}^{-h}\left(\mathrm{~d} h_{2}-h_{2} \mathrm{~d} \log h_{1}\right)=0 \\
\mathrm{~d}\left(\widetilde{\xi}_{n+1}^{++]}+\widetilde{\xi}_{n-1}^{+]}\right)+\left((n+1) \widetilde{\xi}_{n+1}^{++]}-(n-1) \widetilde{\xi}_{n-1}^{++]}\right)\left(\frac{1}{2} \mathrm{~d} h-\mathrm{d} \log h_{1}\right)+n \mathrm{e}^{-h / 2} \widetilde{\xi}_{n}^{++]}\left(\mathrm{d} h_{2}-h_{2} \mathrm{~d} \log h_{1}\right)=0
\end{gathered}
$$

$$
n \geq 2 \text {. }
$$

This recursive system allows in principle to compute all the coefficients in (B2) in terms of the Przanowski function $h$. In particular, the integrability condition for the first equation reduces to the master equation.

${ }^{1}$ A. Besse, Einstein Manifolds (Springer, New York, 1987).

${ }^{2}$ J. Bagger and E. Witten, Nucl. Phys. B 222, 1 (1983).

${ }^{3}$ A. Strominger, Phys. Lett. B 421, 139 (1998); e-print arXiv:hep-th/9706195.

${ }^{4}$ K. Becker and M. Becker, Nucl. Phys. B 551, 102 (1999); e-print arXiv:hep-th/9901126.

${ }^{5}$ M. Gutperle and M. Spalinski, J. High Energy Phys. 2000, 037 (2000); e-print arXiv:hep-th/0005068.

${ }^{6}$ S. V. Ketov, Nucl. Phys. B 649, 365 (2003); e-print arXiv:hep-th/0209003.

${ }^{7}$ I. Antoniadis, R. Minasian, S. Theisen, and P. Vanhove, Class. Quantum Grav. 20, 5079 (2003); e-print arXiv:hep-th/ 0307268.

${ }^{8}$ L. Anguelova, M. Roček, and S. Vandoren, Phys. Rev. D 70, 066001 (2004); e-print arXiv:hep-th/0402132.

${ }^{9}$ M. Davidse, U. Theis, and S. Vandoren, Nucl. Phys. B 697, 48 (2004); e-print arXiv:hep-th/0404147.

${ }^{10}$ M. Davidse, F. Saueressig, U. Theis, and S. Vandoren, J. High Energy Phys. 2005, 065 (2005); e-print arXiv:hep-th/ 0506097.

${ }^{11}$ S. Alexandrov, F. Saueressig, and S. Vandoren, J. High Energy Phys. 2006, 040 (2006); e-print arXiv:hep-th/0606259.

${ }^{12}$ L. Bao, A. Kleinschmidt, B. E. W. Nilsson, D. Persson, and B. Pioline, Commun. Num. Theor. Phys. 4, 187 (2010); e-print arXiv:0909.4299.

${ }^{13}$ M. Przanowski, Acta Physiol. Pol. B14, 625 (1983).

${ }^{14}$ M. Przanowski, J. Math. Phys. 31, 300 (1990).

${ }^{15} \mathrm{P}$. Tod, e-print arXiv:hep-th/0609071.

${ }^{16}$ S. M. Salamon, Ann. Sci. Ec. Normale Super. 19, 31 (1986).

${ }^{17}$ B. de Wit, M. Roček, and S. Vandoren, J. High Energy Phys. 2001, 039 (2001); e-print arXiv:hep-th/0101161.

${ }^{18}$ Observe that, except for the last step, the argument just outlined holds in arbitrary dimension: for $n>1$, it is still possible to parametrize the most general $4 n$-dimensional quaternion-Kähler manifold in Przanowski-type form (9), with $\mathcal{X}_{\mathcal{C}}$ chosen in one's favorite Darboux form such as $\mathcal{X}_{\mathcal{C}}=\sum_{i=1}^{n} z^{2 i-1} \mathrm{~d} z^{2 i}$. However, the constraints of quaternion-Kähler geometry will involve $2 n-1$ partial differential equations rather than a single one.

${ }^{19}$ S. Alexandrov, B. Pioline, F. Saueressig, and S. Vandoren (unpublished).

${ }^{20}$ A. Neitzke, B. Pioline, and S. Vandoren, J. High Energy Phys. 2007, 038 (2007); e-print arXiv:hep-th/0701214.

${ }^{21}$ S. M. Salamon, Invent. Math. 67, 143 (1982).

22 "Pseudo" refers to the fact that for negative scalar curvature $\Lambda<0$, the metric (11) is pseudo-Riemannian with signature $(2,4)$, whereas for $\Lambda>0$ it is positive definite.

${ }^{23}$ S. Alexandrov, B. Pioline, F. Saueressig, and S. Vandoren, J. High Energy Phys. 2009, 044 (2009).

${ }^{24}$ The factor of $2 / t$ in (14) is purely conventional. 
${ }^{25}$ The transition function $H^{[i j]}$ is related to the standard Hamilton function $S^{[i j]}$ via $S^{[i j]}=\alpha^{[j]}+\xi^{[i]} \xi^{[j]}-H^{[i j]}\left(\xi^{[i]}, \xi^{j ; j}, \alpha^{[j]}\right)$. In particular, $H^{[i j]}=0$ for the identity map.

${ }^{26}$ S. Alexandrov, J. Phys. A 42, 335402 (2009).

${ }^{27}$ D. Gaiotto, G. W. Moore, and A. Neitzke, e-print arXiv:0807:4723.

${ }^{28}$ In this subsection we drop the patch index [i], but introduce complex coordinates $u^{i}, i=1,2,3$ on $\mathcal{Z}$, hoping that the use of the same letter will not confuse the reader.

${ }^{29}$ H. Looyestijn and S. Vandoren, J. High Energy Phys. 2008, 024 (2008); e-print arXiv:0801.3949 [hep-th].

${ }^{30}$ M. Roček, C. Vafa, and S. Vandoren, J. High Energy Phys. 2006, 062 (2006); e-print arXiv:hep-th/0512206.

${ }^{31}$ S. Alexandrov, J. High Energy Phys. 2007, 094 (2007); e-print arXiv:hep-th/0702203.

${ }^{32}$ K. P. Tod, Geometry and Physics (Aarhus, 1995), Lecture Notes in Pure and Applied Mathematics Vol. 184 (Dekker, New York, 1997), pp. 307-312.

${ }^{33}$ M. Jimbo and T. Miwa, Publ. Res. Inst. Math. Sci. 19, 943 (1983).

${ }^{34}$ K. Ueno and K. Takasaki, Adv. Stud. Pure Math. 4, 1 (1984).

${ }^{35}$ K. Takasaki and T. Takebe, Rev. Math. Phys. 7, 743 (1995); e-print arXiv:hep-th/9405096.

${ }^{36}$ K. Galicki, Commun. Math. Phys. 108, 117 (1987).

${ }^{37}$ M. Przanowski, J. Math. Phys. 32, 1004 (1991).

${ }^{38}$ D. M. J. Calderbank and H. Pedersen, J. Diff. Geom. 60, 485 (2002).

${ }^{39}$ P.-Y. Casteill, E. Ivanov, and G. Valent, Nucl. Phys. B 627, 403 (2002); e-print arXiv:hep-th/0110280.

${ }^{40}$ S. Alexandrov, B. Pioline, F. Saueressig, and S. Vandoren, Lett. Math. Phys. 87, 225 (2009).

${ }^{41}$ The coordinate $z^{2}$ in (97) is not well defined for $w=0$. One can cure this by defining $z^{1}=z$ and $\tilde{z}^{2}=w / z$. The resulting Przanowski solution in these coordinates then takes the same form as in (98), but now in the tilde variables. On the overlap, where $w \neq 0$, we have that $\vec{z}^{2}=-1 / z^{2}$ and $\tilde{z}^{1}=-z^{1} z^{2}$, so the Przanowski function changes according to (6) with $g\left(z^{2}\right)=-1 / z^{2}$ and $f\left(z^{1}, z^{2}\right)=-z^{1} z^{2}$.

${ }^{42}$ For that one should use the trick explained in Sec. 3.4 of Ref. 40 which allows to close the integration contour despite the presence of the logarithmic cuts. This amounts to integrating the function $H^{\left[0 t_{+}\right]}$around the figure-eight contour surrounding $t_{ \pm}$.

${ }^{43}$ They are related to the coordinates used in Ref. 11 as $\chi=-\zeta / 2, \varphi=\widetilde{\zeta}$, and our $\sigma$ corresponds to $4 \sigma+2 \chi \varphi$ there.

${ }^{44}$ S. Alexandrov and F. Saueressig, J. High Energy Phys. 2009, 108 (2009).

${ }^{45}$ In principle, the patches $\mathcal{U}_{ \pm}$are sufficient in order to cover the twistor space of the perturbative universal hypermultiplet. Nevertheless, it is convenient to introduce the additional patch $\mathcal{U}_{0}$ for reasons explained in Ref. 19.

${ }^{46}$ To simplify the notations, we will omit the index [0] for the twistor lines in this patch.

${ }^{47}$ M. Gunaydin, A. Neitzke, O. Pavlyk, and B. Pioline, Commun. Math. Phys. 283, 169 (2008).

${ }^{48}$ M. A. H. MacCallum and R. Penrose, Phys. Rep. 6, 241 (1972).

${ }^{49}$ M. G. Eastwood, R. Penrose, and R. O. Wells, Commun. Math. Phys. 78, 305 (1981).

${ }^{50}$ In dimension $4 n>4$, Eq. (136), in fact, produces solutions of the stronger condition $\left(\epsilon^{A^{\prime} B^{\prime}} \nabla_{A A^{\prime}}, \nabla_{B B^{\prime}}-\nu \epsilon_{A B}\right) \psi=0$, which implies $[\Delta-R /(2(n+2))] \psi=0$ (Ref. 20).

${ }^{51}$ C. LeBrun and S. Salamon, Invent. Math. 118, 109 (1994).

${ }^{52}$ It is not difficult to generalize (142) to include the variations in anomalous dimensions. In this case the result takes the form $\delta h=-h_{1}\left[\oint(\mathrm{d} t / 2 \pi \mathrm{i} t)\left(H_{(1)}+\delta c_{\tilde{\xi}} \log t \partial_{\tilde{\xi}} H\right)+\left(2+h-\log h_{1}^{2}\right) \delta c_{\alpha}+\left[\left(h_{2}+h_{2}^{-}\right) / 2 h_{1}\right]\left(h-\log h_{1}^{2}\right) \delta c_{\tilde{\xi}}^{\tilde{\xi}}\right]$. Although the anomalous dimension terms are not integrals of a holomorphic function, they are annihilated by the conformal Laplacian.

${ }^{53}$ This fact can be understood heuristically as follows. Open contour integrals are expected to arise from closed contour integrals, associated with standard coverings of $\mathcal{Z}$ by open patches, upon shrinking the integration contour around branch cuts in the transition functions. Since the transition functions are holomorphic sections of some line bundle on $\mathcal{Z}$, the end points of the cuts, and therefore of the open contours, must be given by holomorphic sections $t\left(x^{\mu}\right)$ of $\mathcal{Z}$.

${ }^{54}$ The inclusion of multicoverings turns $\Psi$ into a dilogarithm sum (Ref. 23).

${ }^{55}$ R. Bielawski, e-print arXiv:math.DG/0006142.

${ }^{56}$ M. Dunajski and L. Mason, J. Math. Phys. 44, 3430 (2003).

${ }^{57}$ B. Pioline and S. Vandoren, J. High Energy Phys. 2009, 008 (2009). 